ARTICLE

https://doi.org/10.1038/s41467-019-13408-7

\title{
Release factor-dependent ribosome rescue by BrfA in the Gram-positive bacterium Bacillus subtilis
}

\author{
Naomi Shimokawa-Chiba ${ }^{1,3}$, Claudia Müller ${ }^{2,3}$, Keigo Fujiwara (10 ${ }^{1}$, Bertrand Beckert ${ }^{2}$, Koreaki Ito (D) 1 , \\ Daniel N. Wilson (1) ${ }^{2 \star} \&$ Shinobu Chiba (1) ${ }^{1 \star}$
}

Rescue of the ribosomes from dead-end translation complexes, such as those on truncated (non-stop) mRNA, is essential for the cell. Whereas bacteria use trans-translation for ribosome rescue, some Gram-negative species possess alternative and release factor (RF)dependent rescue factors, which enable an RF to catalyze stop-codon-independent polypeptide release. We now discover that the Gram-positive Bacillus subtilis has an evolutionarily distinct ribosome rescue factor named BrfA. Genetic analysis shows that $B$. subtilis requires the function of either trans-translation or BrfA for growth, even in the absence of proteotoxic stresses. Biochemical and cryo-electron microscopy (cryo-EM) characterization demonstrates that BrfA binds to non-stop stalled ribosomes, recruits homologous RF2, but not RF1, and induces its transition into an open active conformation. Although BrfA is distinct from E. coli ArfA, they use convergent strategies in terms of mode of action and expression regulation, indicating that many bacteria may have evolved as yet unidentified ribosome rescue systems.

\footnotetext{
${ }^{1}$ Faculty of Life Sciences and Institute for Protein Dynamics, Kyoto Sangyo University, Motoyama, Kamigamo, Kita-Ku, Kyoto 603-8555, Japan. ${ }^{2}$ Institute for Biochemistry and Molecular Biology, University of Hamburg, Martin-Luther-King-Platz 6, 20146 Hamburg, Germany. ${ }^{3}$ These authors contributed equally: Naomi Shimokawa-Chiba, Claudia Müller. *email: Daniel.Wilson@chemie.uni-hamburg.de; schiba@cc.kyoto-su.ac.jp
} 
aithful translation requires accurate initiation, elongation, and termination. In translation termination, the stop codon situated in the A-site of the ribosome recruits a release factor (RF), which then hydrolyzes the peptidyl-tRNA ester bond to release the polypeptide product from the ribosome. In bacteria, RF1 recognizes UAA and UAG while RF2 recognizes UAA and UGA through their PxT and SPF codon recognition motifs, respectively ${ }^{1}$. These RFs contain the hydrolysis active site motif, GGQ, for catalysis. Polypeptide release is then followed by dissociation of the ribosome from mRNA into the small and large subunits by a process mediated by ribosome recycling factor and elongation factor $\mathrm{G}^{2}$.

However, the termination/recycling event can be perturbed when mRNA has aberrant features, one of which is the absence of an in-frame stop codon. The mRNA lacking a stop codon, called a non-stop mRNA, causes stalling of the ribosome at the $3^{\prime}$ end because recruitment of RFs to the ribosome requires the interaction of a stop codon recognition motif of the RF with a cognate stop codon. Since the role of termination is not only to define the end of the protein but also to recycle the ribosome for the next round of translation initiation, a failure in termination lowers the cellular capacity of protein synthesis, unless dealt with by the cellular quality control mechanisms. Indeed, a loss of function in the quality control machinery leads to an accumulation of deadend translation products, which was estimated to represent $\sim 2-4 \%$ of the translation products in Escherichia coli $^{3}$, and results in lethality ${ }^{4,5}$.

Living organisms have evolved mechanisms that resolve nonproductive translation complexes produced by ribosome stalling on non-stop mRNAs. Such quality control is also called ribosome rescue. In eukaryotic cells, like yeast, the Dom34/Hbs1 complex, together with Rli1, mediates ribosome rescue on truncated mRNAs ${ }^{4,6,7}$. In bacteria, two distinct mechanisms operate in the resolution of non-stop nascent chain-ribosome complexes, transtranslation and stop-codon-independent peptide release from the ribosome $e^{4,5,8,9}$. The latter mechanism can further be classified into two classes, RF-dependent and RF-independent. The crucial player in trans-translation is the transfer-messenger RNA (tmRNA), which is encoded by ssrA. tmRNA cooperates with SmpB, which mediates ribosomal accommodation of tmRNA at the ribosomal A-site ${ }^{10,11}$. The tmRNA is composed of tRNA- and mRNA-like domains. The former can be charged with alanine, which then accepts the non-stop peptide and is elongated further according to the mRNA-like coding function of tmRNA until the built-in stop codon is reached. The result is the formation of the non-stop polypeptide bearing an extra $s s r A$-encoded sequence (15 amino acids in B. subtilis) and dissociation of the ribosome from the non-stop mRNA. The SsrA tag sequence promotes proteolytic elimination of the non-stop polypeptide via targeting to cellular proteases. Trans-translation is essential for the growth of some bacteria $^{5,12}$. The essentiality lies in the liberation of the ribosome from the non-stop mRNA, but not in proteolytic degradation of the translation products ${ }^{13,14}$. Bacterial species that can survive without trans-translation often possess one or more alternative ribosome rescue factor(s), such as ArfA, ArfB, or ArfT, which are involved in stop-codon-independent cleavage of the non-stop peptidyl-tRNA ${ }^{5}$.

ArfA was identified in $E$. coli by a genetic screening for a mutation showing synthetic lethality with the loss of $s s r A^{15}$. ArfA is an RF-dependent ribosome rescue factor, which recruits RF2, but not RF1, to the non-stop stalled ribosome complexes to induce hydrolysis of the dead-end peptidyl-tRNA ${ }^{16}$. Interestingly, ArfA itself is produced from a non-stop mRNA, such that it is strongly down-regulated by trans-translation in wild-type cells and only induced significantly upon dysfunction of trans-translation. Thus, it has been suggested that tmRNA-SmpB is the primary rescue factor, and the ArfA-RF2 system serves as a backup system ${ }^{17,18}$. ArfB (YaeJ), identified as a multicopy suppressor of the $s s r A / a r f A$ double mutant ${ }^{19,20}$, contains its own GGQ catalytic motif, enabling it to act as an RF-independent ribosome rescue factor ${ }^{21}$. Although the physiological role of ArfB in E. coli is unknown, its homologs are widely distributed among both Gram-positive and -negative bacteria ${ }^{5,20,22}$ as well as eukaryotic mitochondria ${ }^{23}$. ArfT in Francisella tularensis, a member of $\gamma$ proteobacteria that lacks both ArfA and ArfB homologs ${ }^{24}$, is essential in the absence of tmRNA. Like ArfA, ArfT is an RFdependent ribosome rescue factor, although ArfT can function with either RF1 or RF2. Phylogenetic distribution of ArfA is limited to a subset of $\beta$ - and $\gamma$-proteobacteria whereas that of ArfT is limited to a subset of $\gamma$-proteobacteria. To date, RFdependent ribosome rescue factors have only been reported in Gram-negative bacteria.

Bacillus subtilis, a Gram-positive bacterium, can also survive without $s s r A^{25}$, but no alternative factor for ribosome rescue has been reported in this organism, raising the question of whether the ribosome rescue function is non-essential or alternative factors have escaped identification in B. subtilis. In this study, we have addressed this question and identified BrfA (Bacillus ribosome rescue factor $A$; formerly $\mathrm{YqkK}$ ) as a ribosome rescue factor. BrfA has no obvious sequence similarity to other Arf proteins. We show that BrfA is an RF2-dependent ribosome rescue factor, which induces hydrolysis of peptidyl-tRNA in non-stop translation complexes. BrfA is produced naturally from a non-stop mRNA and is thus negatively regulated by trans-translation, revealing a conceptually similar regulatory crosstalk as documented for E. coli ArfA. Lastly, using single-particle cryo-electron microscopy (cryo-EM), we reveal how BrfA recognizes the presence of truncated mRNAs and recruits and stabilizes an open conformation of RF2 to rescue the stalled ribosomes. BrfA uses a mechanism that is similar but distinct from ArfA. Collectively, our findings lead us to suggest that Gram-positive and -negative bacteria have independently acquired their own unique RFdependent ribosome rescue systems equipped with a convergent scheme of regulation.

\section{Results}

BrfA and trans-translation exhibit synthetic lethality. The dispensability of trans-translation in B. subtilis raises the possibility that it contains an alternative ribosome rescue factor. With the reasoning that the loss of such a factor would make the bacterial growth dependent on trans-translation proficiency, we searched for the chromosomal gene knockouts that cause synthetic lethality with the deficiency of trans-translation. We used a strain with chromosomal deletion of $\operatorname{sip} p$, which encodes the trans-translation co-factor but having a plasmid carrying the wild-type $\operatorname{smp} B$ as well as lacZ genes (Fig. 1a). This rescue plasmid was a derivative of pLOSS ${ }^{\star 26}$ driven by a temperature sensitive (Ts) replicon, such that it is lost frequently at high temperature. We prepared chromosomal DNA from a mixture of the BKE library strains, a collection of mutants individually disrupted for the 3968 non-essential B. subtilis genes by replacements with the erythromycin resistance marker $(e r y)^{27}$, and used it to transform (by homologous recombination) the strain for the screening described above. Transformant mixtures were then incubated at $50{ }^{\circ} \mathrm{C}$ to destabilize the rescue plasmid and plated on selective agar containing X-Gal (see Methods). Whereas clones that did not depend on SmpB had segregated out the plasmid and formed white colonies, those requiring SmpB survived only when they had retained the plasmid and formed blue colonies due to the plasmid-encoded $\beta$-galactosidase (Fig. 1a). Among $~ 74,000$ transformants, we picked up 42 blue colonies and determined the 
a

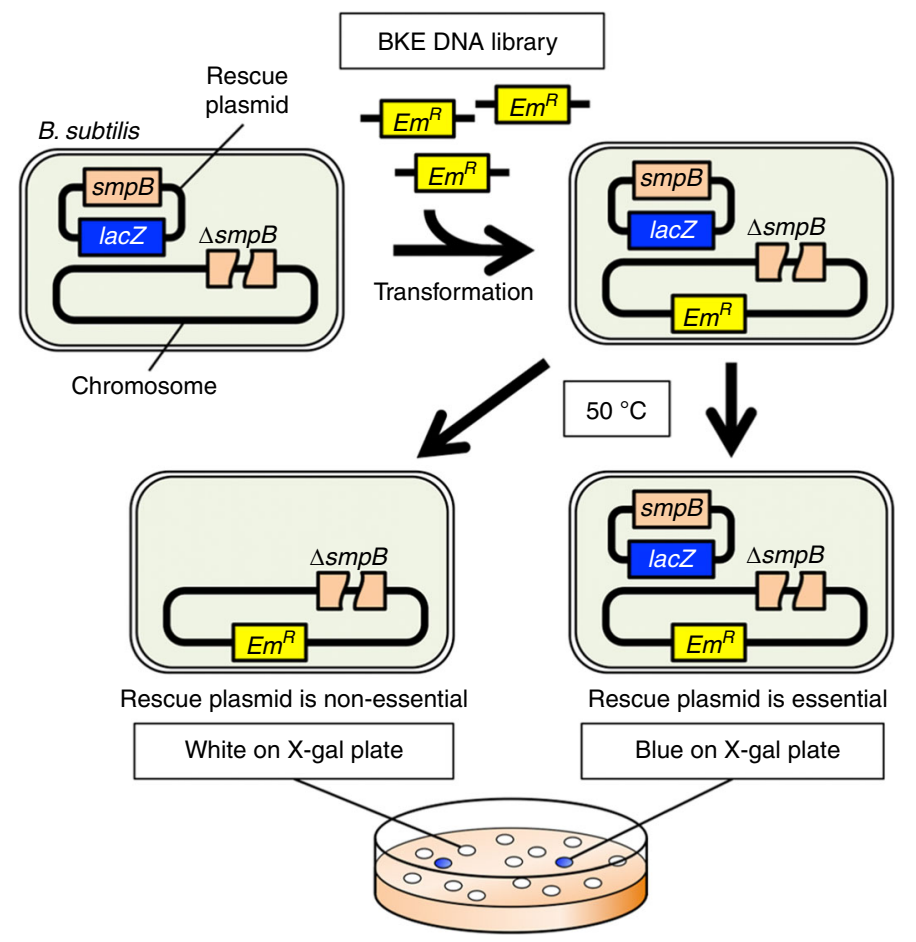

b

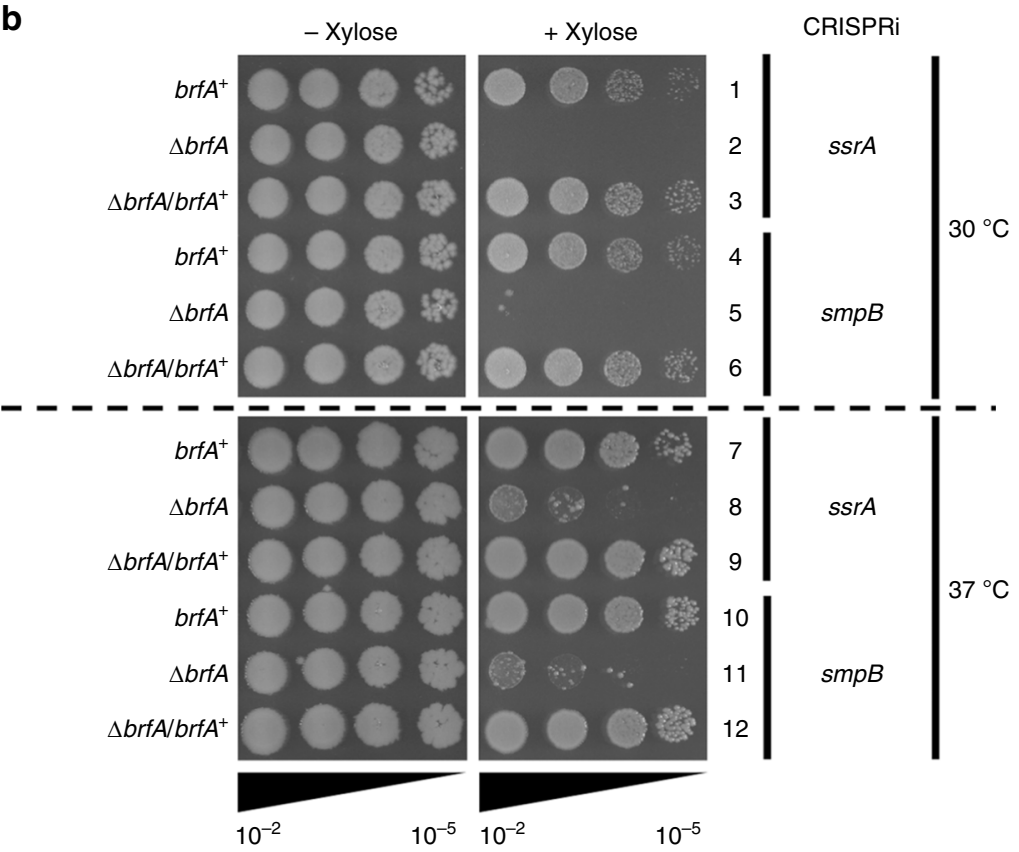

Fig. 1 Simultaneous loss of BrfA and trans-translation leads to synthetic growth defects. a A schematic representation of the synthetic lethal screening to identify genes whose absence causes synthetic lethal phenotype with the deficiency of trans-translation. $\mathbf{b}$ Xylose-inducible CRISPRi was targeted to ssr $A$ (lines 1-3, 7-9) or smpB (lines 4-6, 10-12) in the B. subtilis strains indicated at the left by the genotypes of the brfA gene $\left(\Delta b r f A / b r f A^{+}\right.$signifies the presence of brfA ${ }^{+}$in an ectopic locus). Cultures prepared in the absence of xylose were serially diluted (from $10^{-2}$ to $10^{-5}$ ) and spotted onto $\mathrm{LB}$ agar plates with or without $1 \%$ xylose, as indicated at the top, for incubation at $30^{\circ} \mathrm{C}$ (upper) or $37^{\circ} \mathrm{C}$ (lower) for $17 \mathrm{~h}$.

chromosomal locations of the ery inserts by DNA sequencing, followed by elimination of false-positive clones by retransformation experiments. These procedures left clones with an ery disruption of $y q k K$ (renamed $b r f A$, see below), which makes $\mathrm{SmpB}$ indispensable for survival.

We validated the growth requirement features of B. subtilis for BrfA and the trans-translation system by an independent approach. We used CRISPR interference (CRISPRi) ${ }^{28}$ to conditionally silence either $s s r A$ or $\operatorname{smp} B$ (Fig. 1b). To do this, the catalytically inactive and xylose-inducible variant of Cas9 (dCas9) was integrated into the chromosomal lacA locus of the wild type (WT) and the $\triangle b r f A$ strains. In addition, the constitutively expressed small guide RNA (sgRNA) that targets either $s s r A$ or $\operatorname{smp} B$ was integrated into the amyE locus of the same strains. The bacterial strains grew normally when dCas9 was uninduced in the absence of xylose (Fig. 1b, left panels). Growth of the $b r f A^{+}(\mathrm{WT})$ cells was not affected by $1 \%$ xylose, which induced dCas 9 to silence $\operatorname{ssr} A$ or $\operatorname{smp} B$ (lanes $1,4,7$, and 10, right 
panels). By contrast, growth of the $\triangle b r f A$ strain was severely impaired in the presence of xylose, which led to CRISPRimediated repression of $s s r A$ or $\operatorname{smp} B$ (lanes 2, 5, 8, and 11, right panels). Expression of $b r f A$ from an ectopic locus restored the growth defect associated with the trans-translation deficiency, substantiating that BrfA is the responsible factor for the synthetic growth phenotype (lanes 3, 6, 9, and 12). These results indicate that BrfA is required for optimal growth in the absence of sufficient activity of trans-translation in B. subtilis.

BrfA expression is regulated by trans-translation. The $b r f A$ gene contains 71 sense codons followed by a stop codon. We note that it contains a typical rho-independent transcription terminator sequence within the coding region, raising an intriguing possibility that BrfA is translated naturally from a non-stop mRNA lacking the $3^{\prime}$ region including the stop codon (Fig. 2a). If this is the case, the translation product should initially consist of approximately 62 amino acids in the form of peptidyl-tRNA. However, translation of such mRNAs is likely dealt with by the trans-translation mechanism of ribosome rescue, which would add the SsrA tag sequence of 15 amino acids to the BrfA non-stop product. It follows then that the BrfA product would be rapidly degraded by cellular proteases, such that the protein level should be strongly down-regulated in trans-translation proficient cells.

To test this possibility, we examined the impact of transtranslation on the accumulation of a series of BrfA-derived proteins. We constructed a translational gene fusion consisting of the coding sequences for green fluorescence protein (GFP), BrfA (full length), and FLAG that are connected in-frame in this order from the $\mathrm{N}$-terminus to the $\mathrm{C}$-terminus. We also constructed transcriptional fusions, in which independently translatable lac $Z$ follows $g f p$-brfA-flag to verify the occurrence of transcription termination in brfA (Fig. 2b). We expressed GFP-BrfA-FLAG in the wild type and the $\triangle s m p B$ cells and detected the products with anti-GFP and anti-FLAG immunoblotting. Strikingly, the wildtype cells did not produce any anti-GFP detectable protein from the $g f p$-brfA(WT)-flag construct (Fig. 2c, lane 1, upper panel). By contrast, the $\triangle \sin p B$ cells produced a product that formed an intense band near the $37 \mathrm{kDa}$ marker (Fig. 2c, lane 2, upper panel). This species of protein did not react with anti-FLAG (lane 2, lower panel), indicating that it lacked the C-terminal region. These results are consistent with the notion that the mRNA ends within $\operatorname{brfA}$ due to internal termination. The translation products are SsrA-tagged and degraded rapidly in the wild-type strain, while they accumulate in the trans-translation-deficient $\triangle s m p B$ cells.

To examine the above scenario further, we disrupted the transcription terminator either by internal deletion or by synonymous substitutions (Fig. 2a, b). In the former, we deleted the codons 63-71 of brfA in the context of GFP-BrfA-FLAG to eliminate the $3^{\prime} \mathrm{T}$ stretch of the transcriptional terminator (GFPBrfA62-FLAG). In the latter, we introduced synonymous mutations to disrupt the secondary structure required for transcription termination (GFP-BrfA(no_term)-FLAG) (Fig. 2a, b). Both of these terminator-less mutant forms of GFP-BrfA-FLAG accumulated equally in the wild type and $\triangle \operatorname{sip} B$ strains and are reactive with both anti-GFP and anti-FLAG (Fig. 2c, lanes 3-6).

We then confirmed the occurrence of internal transcription termination by examining the expression of lac $Z$ attached at a $3^{\prime}$ region of the transcription unit. If the terminator within $\operatorname{brf} A$ indeed functions, the downstream lac $Z$ would not be expressed. As expected, cells harboring the construct with the wild-type brfA sequence exhibited very low levels of $\beta$-galactosidase activity (Fig. 2d, columns 1 and 2), as compared with those having the terminator mutations, which exhibited high levels of $\beta$-galactosidase activity (Fig. 2d, columns 3-6). These data establish that the internal transcriptional termination indeed occurs for $\operatorname{brfA}$, indicating that it is a major element that triggers the BrfA down-regulation by the SsrA tag-dependent degradation.

These results show that $B$. subtilis is equipped with at least two layers of ribosome rescue mechanisms, trans-translation and BrfA-dependent peptidyl-tRNA hydrolysis (see below). The trans-translation-dependent down-regulation indicates that BrfA is the secondary ribosome rescue factor that is only produced upon dysfunction of trans-translation, the primary ribosome rescue mechanism. Thus, the internal transcription terminator in BrfA provides the means for this bacterium to accomplish the compensatory and vectorial regulation for the maintenance of ribosome rescue capability. In this context, BrfA bears a striking similarity to the E. coli alternative rescue factor, ArfA, which is also synthesized from a non-stop mRNA ${ }^{17,18}$.

BrfA recruits RF2 to hydrolyze non-stop peptidyl-tRNAs. We characterized BrfA biochemically by examining whether it could induce polypeptide release, as expected for an alternative ribosome rescue factor. To do this, we purified BrfA in the form of BrfA62-His $_{6}$, which lacks the C-terminal 9 amino acid residues encoded by the $b r f A$ gene but not by the $\operatorname{brf} A$ mRNA (see above); a hexahistidine ( $\mathrm{His}_{6}$ ) tag was attached to the $\mathrm{C}$-terminus to aid purification. We considered that the absence of the C-terminal 9 amino acids was physiological. Also, the absence of the $3^{\prime}$ coding region of $b r f A$ should disrupt the internal transcription terminator signal and enable the attachment of the $\mathrm{His}_{6}$ tag. Since BrfA lacks a GGQ motif critical for peptidyl-tRNA hydrolysis, we hypothesized that BrfA requires an RF to hydrolyze peptidyltRNA. Therefore, we also purified B. subtilis RF1 and RF2. For in vitro translation with defined translation components, we used the $B s$ hybrid PURE system ${ }^{29}$, a modified version of the PURE coupled transcription-translation system ${ }^{30}$, in which the original $E$. coli ribosomes were replaced with $B$. subtilis ribosomes. We omitted RFs in the Bs hybrid PURE system unless otherwise stated.

We used DNA fragments encoding GFP but without an inframe stop codon (GFP-ns) to direct in vitro transcription and translation with Bs hybrid PURE system and separated the translation products by neutral $\mathrm{pH}$ SDS-PAGE, which preserved the peptidyl-tRNA ester bond ${ }^{31}$. The major product, migrating between the 42 and the $55 \mathrm{kDa}$ markers, represents the peptidyltRNA (GFP-tRNA; Fig. 3a, lane 1), as treatment of the sample with RNase A before electrophoresis down-shifted this band to the position near the $28 \mathrm{kDa}$ marker, indicative of tRNA removal (GFP; lane 2). Thus, the non-stop template indeed produced a translation-arrested state of ribosome-nascent chain complex, which was unaffected when the reaction mixture included $B$. subtilis RF1 or RF2 (lanes 3-6), as expected from the absence of a stop codon in the template. We then addressed the effects of BrfA. Neither BrfA by itself nor its combination with B. subtilis RF1 affected the production of GFP-tRNA (lanes 7-10). By contrast, the addition of both BrfA and B. subtilis RF2 to the Bs hybrid PURE system resulted in the production of the hydrolyzed GFP band with a concomitant decrease in the level of GFP-tRNA (lanes 11 and 12). Thus, BrfA and RF2 cooperatively catalyze peptide release from the non-stop stalled ribosome.

Distinct requirements of RF2 for rescue and termination. Given that BrfA and RF2 cooperatively induce hydrolysis of nonstop peptidyl-tRNA, the role of RF2 would be to execute the catalysis. Consistent with this expectation, a catalytically inactive RF2 variant, RF2(GAQ), whose GGQ active site had been mutated to GAQ, no longer stimulated hydrolysis of GFP-tRNA 
a

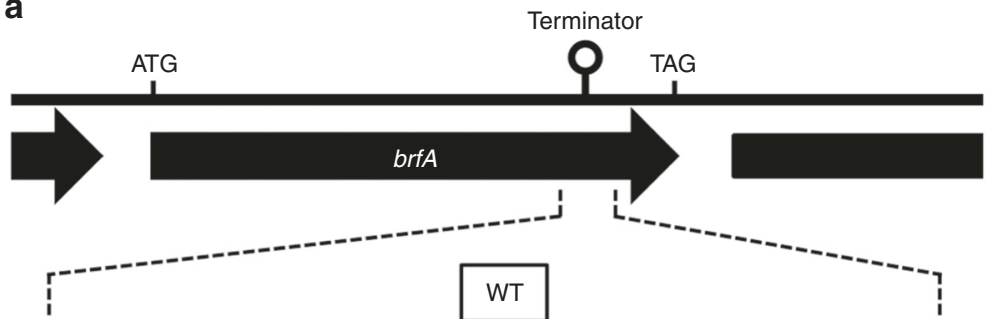

... AagaAuccuUaugaucauacagcaguUgaugauaAggauUuUUUugug . . .

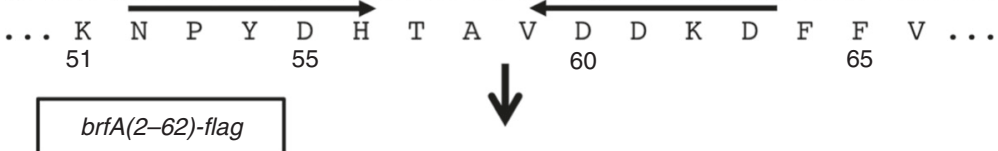

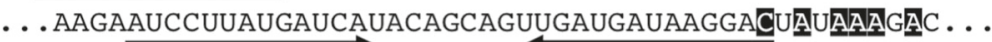

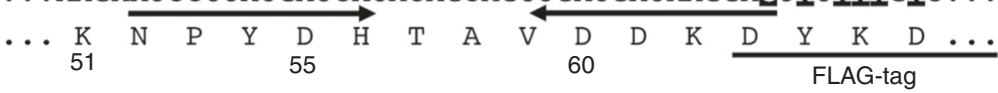



b

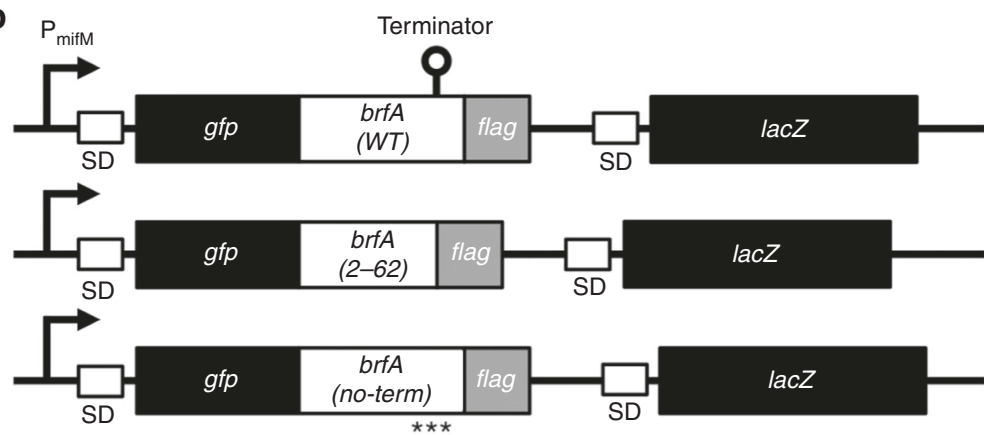

C

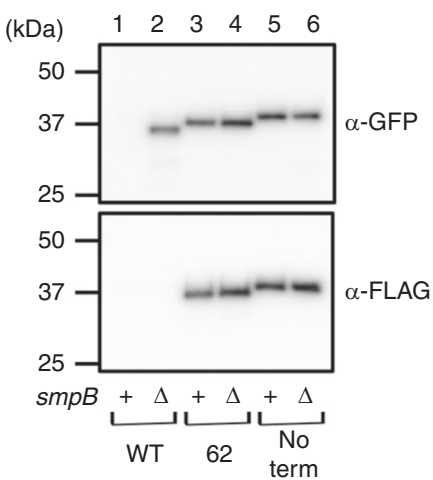

d

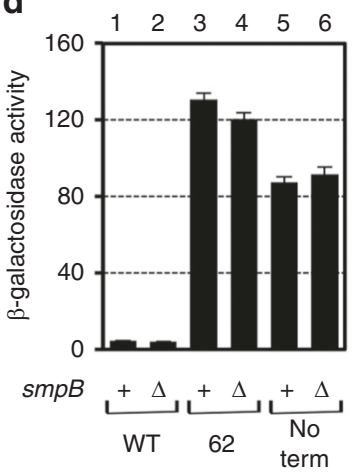

Fig. 2 The cellular abundance of BrfA is negatively regulated by trans-translation. a A schematic representation of the brfA open reading frame, showing the intrinsic transcriptional terminator sequence within the coding region (indicated by "terminator" at the top and the wild type (WT) enlarged view below). Shown also are the corresponding sequences of the gfp-brfA(2-62)-flag and gfp-brfA(no_term)-flag constructs (see b), in which nucleotide substitutions and the FLAG tag are indicated by reverse and underline, respectively. b Schematic representations of the gfp-brfA-flag constructs with wild-type terminator sequence ( $g f p$-brfA(2-71)-flag) and its derivatives with defective terminator signals. These $g f p$-brfA derivatives were placed under the constitutive mif $M$ promoter. The lacZ gene is also placed downstream of the gfp-brfA derivatives. "SD" and asterisks indicate Shine-Dalgarno sequence and synonymous mutations, respectively. c Cellular accumulation of the products of the wild-type construct (lanes 1, 2) as well as the brfA(2-62) (lanes 3, 4) and the brfA (no_term) (lanes 5,6$)$ constructs. They were expressed in the $s m p B^{+}$(odd numbers) or the $\Delta s m p B$ (even numbers) strains and analyzed by anti-GFP (upper) or anti-FLAG (lower) immunoblotting. $\mathbf{d} \beta$-Galactosidase activities (mean \pm s.d., $n=3$ ) of the cells horbaring lac $Z$ at the downstream of wild type (columns 1, 2), the brfA(2-62) (columns 3,4), or the brfA(no_term) (columns 5,6) derivatives of the gfp-brfA in the presence (odd numbers) or absence (even numbers) of $s m p B$. "s.d." indicates standard deviation. Source data are provided as a Source Data file. 
a

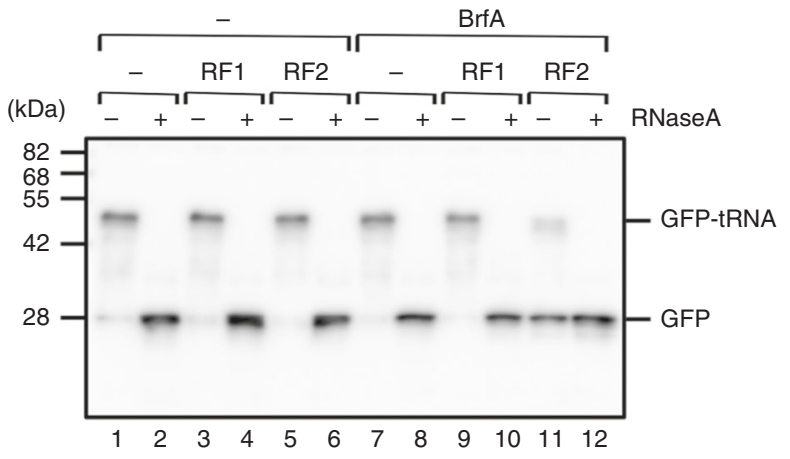

b
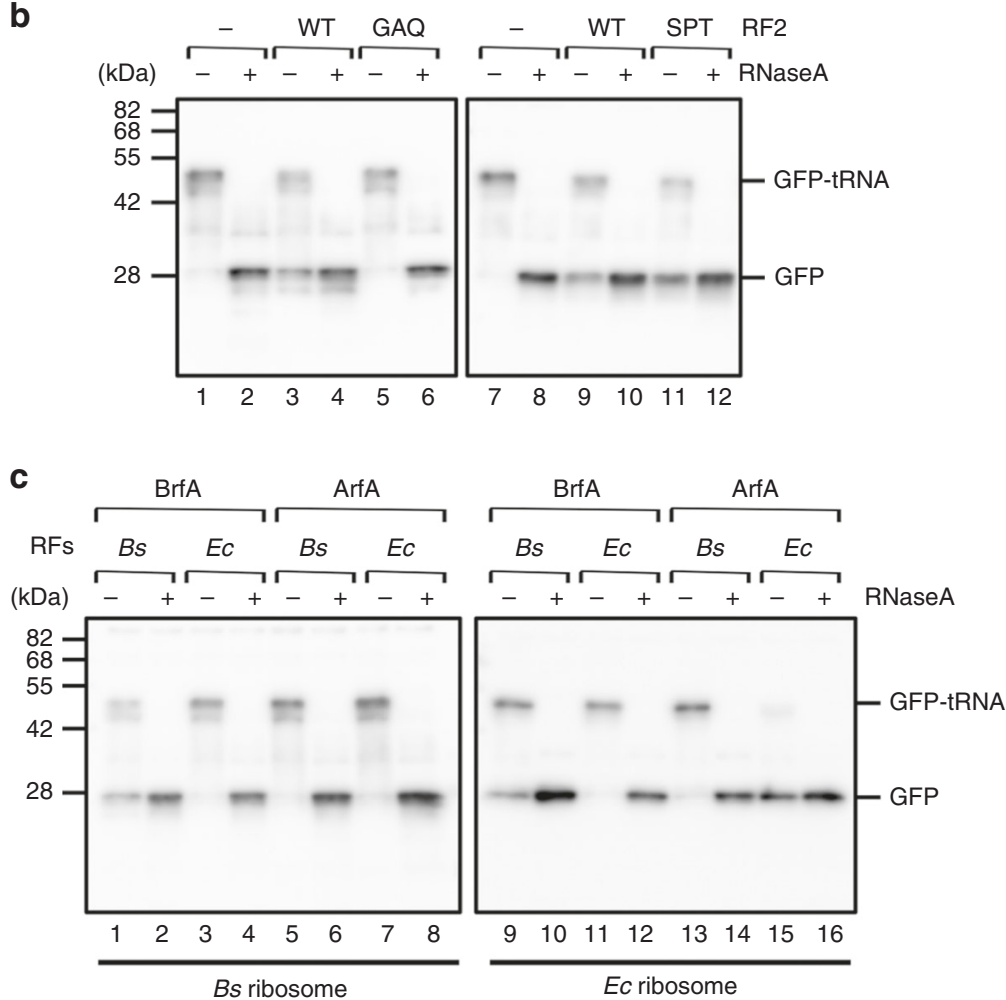

Fig. 3 In vitro activity of BrfA to hydrolyze non-stop peptidyl-tRNA. a BrfA in combination with RF2 cleaves the GFP-tRNA non-stop translation product. In vitro translation using Bs hybrid PURE system was directed by the gfp-ns template. The reaction mixtures contained purified BrfA62-His 6 (lanes 7-12), purified $B$. subtilis RF1 (lanes 3, 4, 9, and 10), and RF2 (lanes 5, 6, 11, and 12), as indicated. Translation was allowed to proceed at $37^{\circ} \mathrm{C}$ for 20 min, and the products were divided into two parts, one of which was treated with RNase A, as indicated. Samples were then analyzed by SDS-PAGE under neutral pH conditions, followed by anti-GFP immunoblotting. b BrfA-dependent peptidyl-tRNA hydrolysis activity of RF2 requires its GGQ active site but not SPF stop codon recognition motif. In vitro translation using Bs hybrid PURE system was directed by the gfp-ns template in the presence of combinations of BrfA, wildtype RF2 (lanes 3, 4, 9, and 10), RF2(GAQ) (lanes 5 and 6), and RF(SPT) (lanes 11 and 12), as indicated. The translation products were analyzed by antiGFP immunoblotting as described above. $\mathbf{c}$ Interspecies compatibility of the RF-dependent rescue factor functions. In vitro translation of $g f p-n s$ was carried out using Bs hybrid PURE system (lanes 1-8) or EC PURE system (lanes 9-16) in the presence of combinations of purified BrfA (lanes 1-4, 9-12), E. coli ArfA (lanes 5-8, 13-16), RFs (RF1 plus RF2) purified from B. subtilis (lanes 1, 2, 5, 6, 9, 10, 13, and 14), and RFs purified from E. coli (lanes 3, 4, 7, 8, 11, 12, 15, and 16). The translation products were analyzed by anti-GFP immunoblotting as described above. Source data are provided as a Source Data file.

even in the presence of BrfA (Fig. 3b, lanes 5 and 6). RF2 possesses the conserved SPF motif as an essential element for the stop codon recognition in termination ${ }^{32}$. We addressed whether this motif is required for the ribosome rescue function of RF2 by mutating it to SPT, which abolishes the termination activity ${ }^{32}$. In the in vitro peptidyl-tRNA hydrolysis assay, the RF2(SPT) was as active as the wild-type RF2 in the BrfA-dependent cleavage of GFP-tRNA (Fig. 3b, lanes 9-12), in comparison with the parallel control reaction without RF2 (lanes 7 and 8). These results demonstrate that the stop codon recognition motif of RF2 is dispensable for the ribosome rescue function.
Ribosome stalling can also be induced by specific amino acid sequences of nascent polypeptides for regulatory purposes. Such regulatory nascent polypeptides include E. coli SecM, B. subtilis MifM, and Vibrio alginolyticus $\mathrm{VemP}^{33,34}$. Ribosome stalling in these cases needs to be adequately regulated, such that it is subject to conditional and specific mechanisms of cancellation ${ }^{35,36}$. Unregulated rescue could be counterproductive in these cases. Our in vivo and in vitro analyses show that the B. subtilis MifM stalling is refractory to both the trans-translation and the BrfA mechanisms of ribosome rescue (Supplementary Fig. 1). Thus, we conclude that BrfA is an RF-dependent ribosome rescue factor in 
B. subtilis that rescues stalling on non-stop mRNAs, but not regulatory stalling peptides, leading us to suggest the renaming of YqkK to BrfA (Bacillus ribosome rescue factor $A$ ). To our knowledge, RF-dependent ribosome rescue factors have not been reported previously in Gram-positive bacteria.

Low interspecies compatibility of ribosome rescue systems. BrfA homologs are conserved among a subset of Bacillaceae family members, mainly among those belonging to the Bacillus genus. Although we do not rule out the possibility that BrfA and ArfA share the same evolutionary origin, their distinct phylogenetic distributions and the unique sequence features (Supplementary Fig. 2) seem to favor the notion that BrfA and ArfA have evolved independently of each other and independently from ArfT and ArfB. The narrow phylogenetic distributions of the RFdependent rescue factors also implies that they emerged relatively late in evolution. Translation components are largely conserved across the species, but they have undergone some micro diversifications. If the alternative rescue factors evolved more recently than the translation factors, their interactions with the translation components, such as the ribosome and an RF, might be speciesspecific. Indeed, Francisella tularensis ArfT can work with F. tularensis RF1 or RF2, but not E. coli $\mathrm{RFs}^{24}$. Also, E. coli ArfA fails to recruit Thermus thermophilus RF2 (refs. ${ }^{37,38}$ ). We tested the compatibility of B. subtilis BrfA and E. coli ArfA with heterologous RFs in vitro. While BrfA efficiently hydrolyzed GFPtRNA in the Bs PURE system supplemented with RF1 and RF2 of B. subtilis (Fig. 3c, lanes 1 and 2), it did not work with the E. coli RFs (lanes 3 and 4). Interestingly, BrfA was functional when the substrate was translated by the E. coli ribosome, provided that the $B$. subtilis RFs were available (lanes 9-10). These results suggest that BrfA interaction with RF2 is species-specific, but its interaction with the ribosome is rather promiscuous.

By contrast, we could show that E. coli ArfA requires both the RF2 as well as the ribosomes to be derived from E. coli. Specifically, ArfA did not work if combined with the E. coli RF2 when the substrate was translated by the B. subtilis ribosomes (Fig. 3c, lanes 7 and 8), nor did it work with B. subtilis RF2 (lanes 5 and 6), even if the substrate was translated by $E$. coli ribosomes (lanes 13 and 14). The E. coli ArfA-mediated hydrolysis of GFPtRNA was only observed when the ribosomes and RFs were both derived from E. coli (lanes 15 and 16). Thus, E. coli ArfA is incompatible with the B. subtilis ribosomes and RF2, analogously to what was previously observed between E. coli ArfA and T. thermophilus RF2 (refs. ${ }^{37,38}$ ). The high specificity of molecular interactions involving the RF-dependent rescue factors is in contrast to the broader interactions in the tmRNA- and ArfBbased rescue pathways (see Discussion).

Cryo-EM structure of a BrfA-RF2-non-stop-ribosome complex. Since B. subtilis BrfA and RF2 can rescue E. coli ribosomes stalled on truncated non-stop mRNAs (Fig. 3c, Supplementary Fig. 3), we formed BrfA-RF2-non-stop 70S ribosome (ns70S) complexes by incubating B. subtilis BrfA and RF2 with E. coli ns70S complexes used previously for ArfA ${ }^{39}$. By substituting the wild-type $B$. subtilis RF2 with a catalytically inactive GGP mutant ${ }^{40,41}$, peptidyl-tRNA hydrolysis and therefore recycling of the ns70S complex was prevented (Supplementary Fig. 3). Cryo-EM analysis of the BrfA62His-RF2-GGP-ns70S complex (herein referred as BrfA-RF2-ns70S) and extensive in silico sorting of this dataset yielded a major subpopulation of ribosomal particles $(>80 \%)$ that contained stoichiometric occupancy of BrfA, RF2 and P-site tRNA (Supplementary Fig. 4). Refinement of this subpopulation led to a final cryo-EM reconstruction of the BrfA-RF2-ns70S (Fig. 4a), with an average resolution of $3.06 \AA$ (Supplementary
Fig. 4, Supplementary Table 1). The cryo-EM density for BrfA was well-resolved with local resolution ranging between $3.0-3.6 \AA$ (Fig. 4b), enabling residues $2-55$ of BrfA to be modeled de novo (Fig. 4c, d). BrfA contains an N-terminal $\alpha$-helix $\alpha 1$ (residues 4-17) followed by a short $\alpha$-helical turn ( $\alpha 2$, residues $21-25)$ and $\beta$-strand ( $\beta 1$, residues $35-38)$ as well as a short C-terminal $\alpha$-helix $(\alpha 3,40-47)$ followed by a positively charged region (residues 48-55) (Fig. 4d).

Interaction of BrfA with the non-stop $70 \mathrm{~S}$ ribosome. The binding site of BrfA is located predominantly on the $30 \mathrm{~S}$ subunit in the vicinity of the decoding center, where it spans from the top of helix 44 (h44) of the 16S rRNA past the ribosomal protein uS12 and reaches into the mRNA channel formed by the head and body of the $30 \mathrm{~S}$ (Fig. 4a). The overall binding site of BrfA on the ribosome (Fig. 4a) is similar, but distinct, to that observed previously for $E$. coli ArfA $37-39,42-44$. The N-terminal helix al of BrfA resides within the intersubunit space, where highly conserved charged residues (Supplementary Fig. 2) establish interactions with the major groove of $\mathrm{h} 44$ and the minor groove of $\mathrm{H} 71$ of the $23 \mathrm{~S}$ rRNA (Fig. 4e). In addition, Arg25 within helix $\alpha 2$ of BrfA, which is conserved in all BrfA sequences (Supplementary Fig. 2), stacks upon U1915 and flips C1914 out of H69, where it stacks upon His133 of RF2 (Fig. 4f). This contrasts with the canonical conformation of $\mathrm{C} 1914$ within $\mathrm{H} 69$ that is observed during translation termination (Fig. 4g) as well as ArfA-mediated ribosome rescue. Indeed, these N-terminal $\alpha$-helices have no counterpart in ArfA, instead the N-terminus of ArfA is unstructured and folds back to interact with uS12 (refs. ${ }^{37-39,42-44}$ ) (Supplementary Fig. 5a-c).

The C-terminal region of BrfA extends from the decoding center into the mRNA channel and would be incompatible with the presence of a full-length mRNA (Fig. 4h), but compatible with a truncated non-stop mRNA (Fig. 4i). BrfA exhibits a modest overlap with the second $(+2)$ and third $(+3)$ nucleotide of the Asite codon, but extensive steric clashes would be expected for the subsequent positions ( +4 onwards) (Fig. 4i), similar to that observed previously for ArfA $37-39,42-44$ (Supplementary Fig. 5d-i). Thus, BrfA may also recycle ribosomes stalled on non-stop mRNAs with 1-3 nucleotides extending into the A-site, as shown experimentally for ArfA ${ }^{45-47}$. The positively charged C-terminus of BrfA can form multiple hydrogen bond interactions with $16 \mathrm{~S}$ rRNA nucleotides that comprise the mRNA channel (Fig. 4j). While the interaction network is generally distinct from that observed for ArfA, we note that the mode of contact between the side chains of Lys49 and His50 of BrfA with U534 of the 16S rRNA appears to be shared by $\operatorname{ArfA}^{37-39,42-44}$ (Supplementary Fig. $5 \mathrm{j}-1)$.

BrfA stabilizes an open conformation of RF2 on the ribosome. The structure of the BrfA-RF2-ns70S reveals that BrfA recruits RF2 by establishing an extensive interaction surface, specifically encompassing the central portion (residues 30-40) of BrfA and domain 2 (d2) of RF2 (Fig. 5a). Similar to ArfA ${ }^{37-39,42-44}$ (Supplementary Fig. 6A-C), BrfA also donates the small $\beta$-strand $(\beta 1)$ to augment the $\beta$-sheet of the superdomain $\mathrm{d} 2 / \mathrm{d} 4$ of RF2 (Fig. 5a). The overall position of RF2 in BrfA-RF2-ns70S is similar, but slightly shifted, compared to that observed during canonical translation termination ${ }^{40,48}$ (Fig. 5b, Supplementary Fig. 6d-f). The shift is larger and more global than reported previously for ArfA $37-39,42-44$ (Supplementary Fig. 6a-c), which may arise in part due to the differences between $B$. subtilis and E. coli RF2s. The shift affects the loop between the $\beta 4$ and $\beta 5$ strands of $\mathrm{d} 2$ bearing the SPF (B. subtilis 202-Ser-Pro-Phe204) motif, which is involved in the specificity of recognition of the first and second positions of UGA/UAA stop codons $32,40,48$ 
a

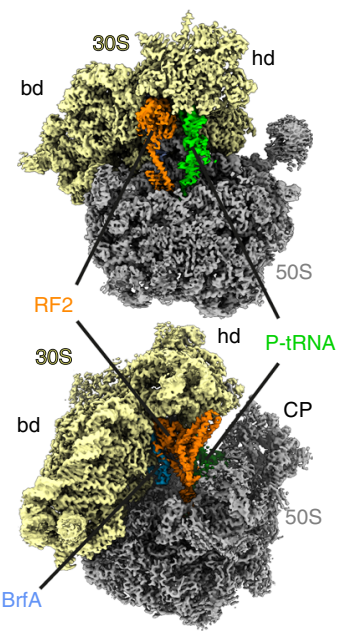

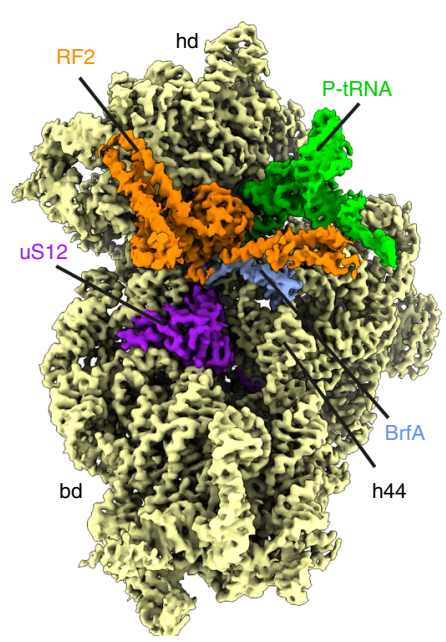

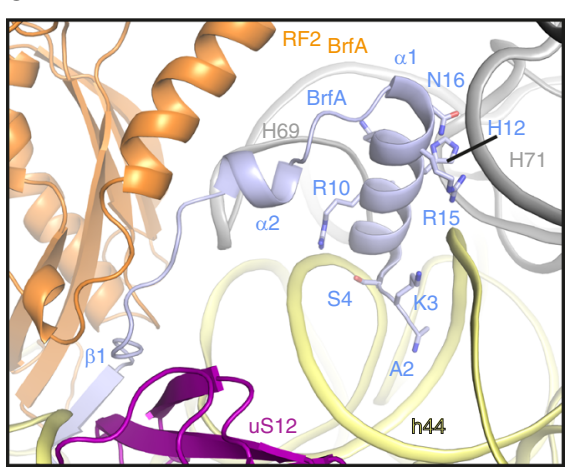

h

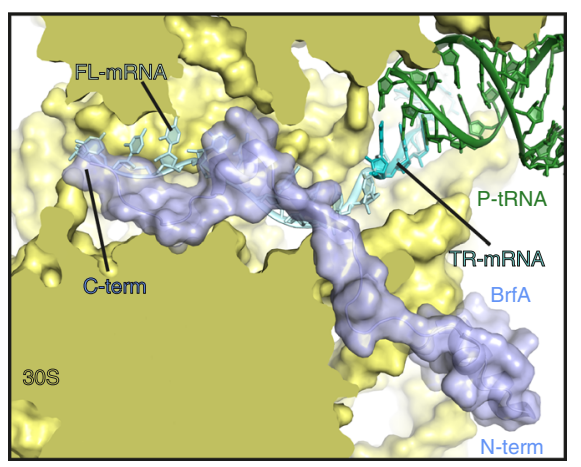

f

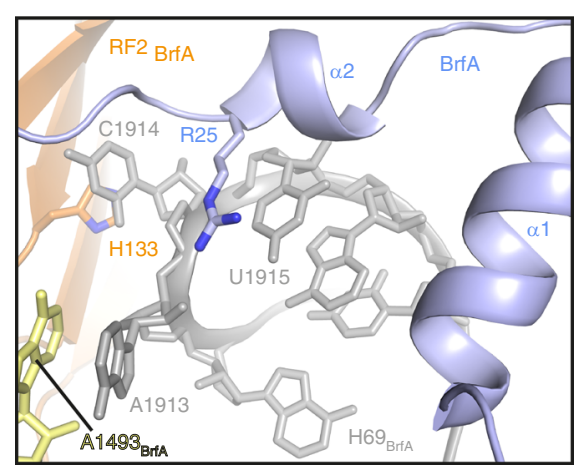

i

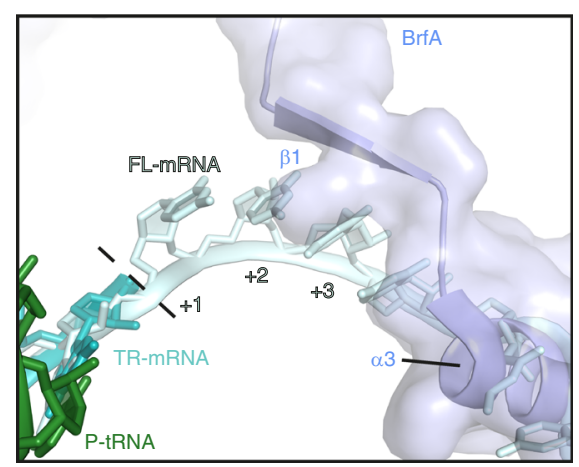

BrfA 1-62

\begin{tabular}{|l|l|l|l|l|l|l|l|l|l|l|l|}
\hline & 4 & & 17 & 21 & 25 & 35 & 38 & 40 & 47 & 55 & \\
\hline & & $\alpha 1$ & & $\alpha 2$ & & $\beta 1$ & & $\alpha 3$ & & not modelled \\
\hline
\end{tabular}

b

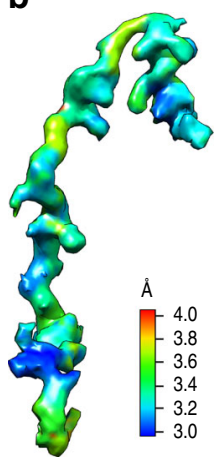

C

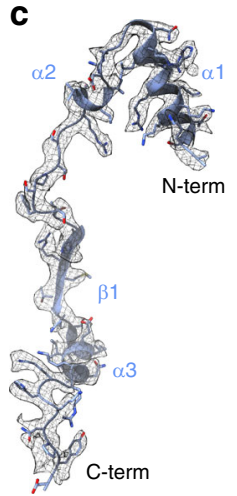

d

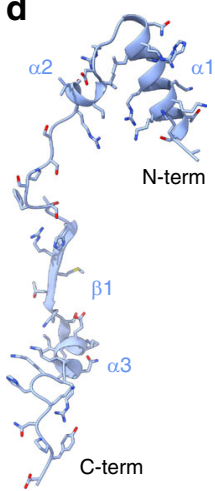

g

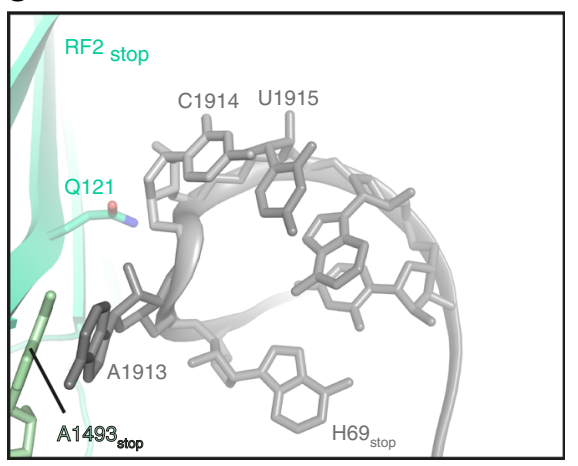

j

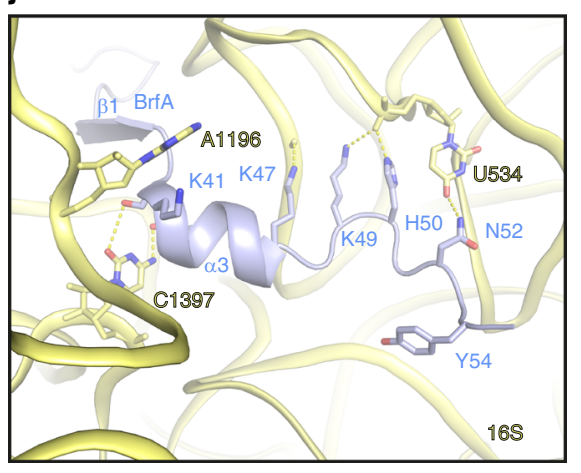

Fig. 4 Cryo-EM structure of BrfA-RF2-ns70S complex. a Different views of the cryo-EM map of the BrfA-RF2-ns70S complex with isolated densities highlighting the $30 \mathrm{~S}$ (yellow: hd, head; bd, body) and $50 \mathrm{~S}$ (gray: CP, central protuberance) subunits, P-site tRNA (green), RF2 (orange), and BrfA (blue). b, c lsolated electron density for BrfA (b) colored according to local resolution and (c) shown as mesh (gray) with fitted molecular model for BrfA. $\mathbf{d}$ Model for BrfA with features highlighted corresponding to the schematic of BrfA protein, including $\alpha$-helical and $\beta$-strand regions. e The $N$-terminus of BrfA (blue) interacts both h44 of the $16 \mathrm{~S}$ rRNA (yellow) and H69 and H71 of the 23S rRNA (gray). f The conserved R25 of BrfA (blue) stacks upon U1915 and causes C1914 to flip out and stack upon H133 of RF2. $\mathbf{g}$ Same view as $\mathbf{f}$ but showing the RF2 ${ }_{\text {stop }}$ (lime) and the conformation of H69 for a canonical termination complex (PDB ID 4V5E48). $\mathbf{h}$ Transverse section of the $30 \mathrm{~S}$ subunit (yellow) to reveal the mRNA channel showing a superimposition of full-length mRNA (FL-mRNA, cyan) with truncated non-stop mRNA (TR-mRNA, teal), P-site tRNA (green), and surface representations of BrfA (blue). i Superimposition of FL-mRNA (cyan) with TR-mRNA (teal), P-site tRNA (green), and transparent surface representation of BrfA (blue). The first (+1), second (+2), and third $(+3)$ nucleotides of the A-site codon of the FL-mRNA are indicated. $\mathbf{j}$ Interaction of the C-terminus of BrfA (blue) with the 16S rRNA showing potential hydrogen bonds with yellow dashed lines.

(Fig. 5c). Importantly, the structure illustrates that BrfA, like ArfA, does not interact with the SPF motif and therefore does not directly mimic the presence of a stop codon (Fig. 5c, Supplementary Fig. $6 \mathrm{~g}-\mathrm{i})$, which is consistent with our observation that mutations in the SPF motif that impair RF2 termination activity do not affect BrfA-RF2-mediated ribosome rescue (Fig. 3b).
During canonical termination, recognition of the stop codon by RF2 (and RF1) is proposed to stabilize a distinct conformation of the switch loop that directs domain 3 into the $\mathrm{PTC}^{1,49}$. The switch loop conformation is stabilized by stacking interactions between Trp318 (B. subtilis numbering) of RF2 and A1492 (h44) as well as between A1493 (h44) and A1913 in H69 (refs. ${ }^{40,48}$ ) 
a

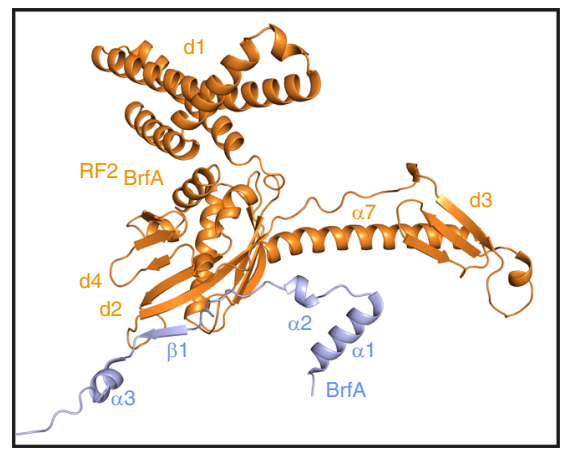

d

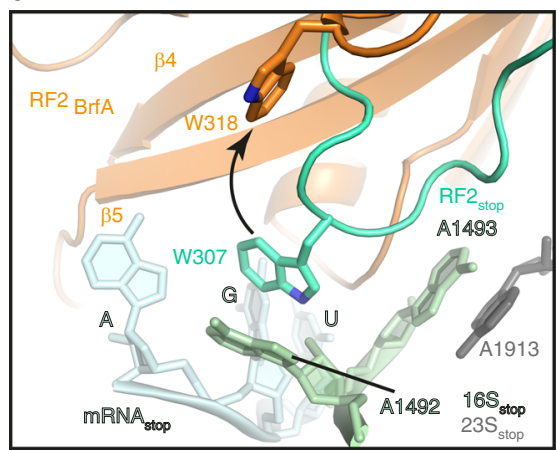

b

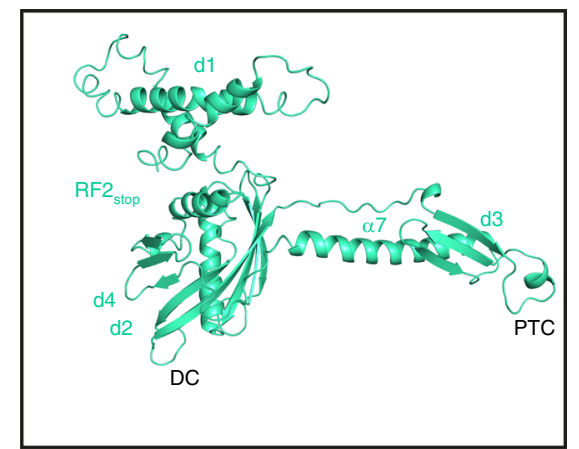

e

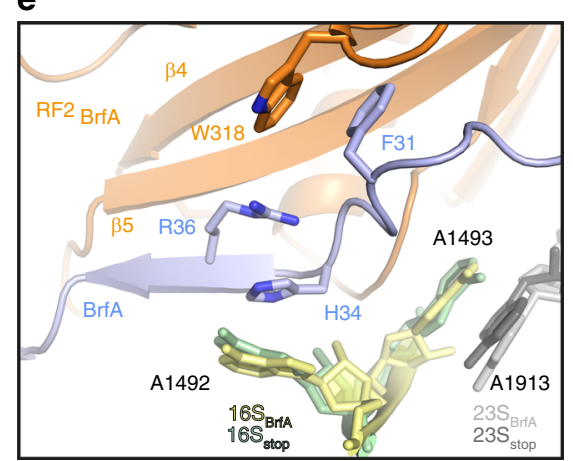

C

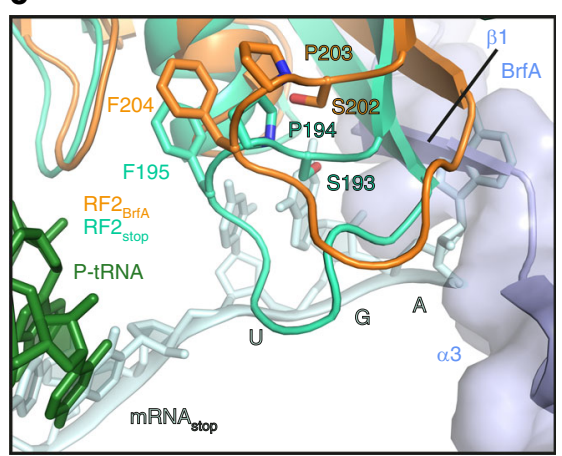

f

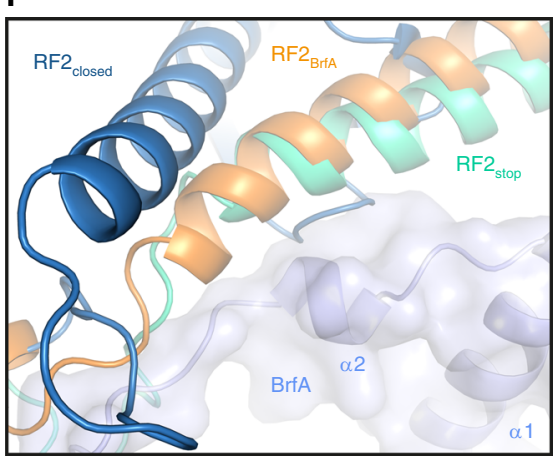

Fig. 5 BrfA recruits and stabilizes the open conformation of RF2 on the ribosome. a, b Overview of the interaction of BrfA (blue) with (a) RF2 (RF2 BrfA, orange) from the BrfA-RF2-ns70S and with (b) RF2 (RF2 stop, lime, PDB ID 4V5E) from a canonical termination complex ${ }^{48}$. RF2 domains 1-4 (d1-d4) and relative positions of the decoding center (DC) and peptidyltransferase center (PTC) are indicated. c Comparison of the relative positions of the SPF motif of RF2 $2_{\text {stop }}$ (lime) and RF2 $2_{\text {Brf }}$ (orange) with BrfA (blue), P-site tRNA (green), and truncated non-stop mRNA (TR-mRNA, cyan) shown for reference. $\mathbf{d}$

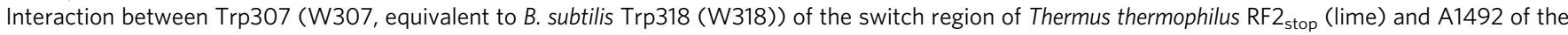

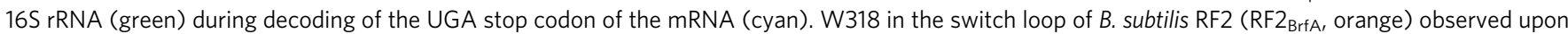
BrfA binding is superimposed and arrowed. e Same view as in d, but showing the conformation of the switch loop of RF2 $\mathrm{BrfA}$ (orange) and A1492/A1493 (yellow) when BrfA (blue) is present. $\mathbf{f}$ Superimposition of the conformation of $\alpha$-helix $\alpha 7$ of RF2 from the crystal structure of the closed form of RF2 (RF2 $2_{\text {closedi }}$ dark blue, PDB ID 1GQE) with RF2 $2_{\text {stop }}$ (lime) and RF2 $2_{\text {BrfA }}$ (orange), with BrfA (blue) shown for reference.

(Fig. 5d). In the BrfA-RF2-ns70S, the presence of BrfA precludes a direct interaction between Trp318 and A1492 (Fig. 5e). Rather, BrfA appears to stabilize a similar conformation of A1492 through stacking interactions with His34 and Arg36 (Fig. 5e), whereas a completely distinct conformation of A1492 (and A1493) is adopted in the presence of ArfA (Supplementary Fig. $7 \mathrm{a}-\mathrm{c}$ ). Additional stacking interactions are also observed between Phe 31 of BrfA and Trp318 within the switch region of RF2 (Fig. 5e), which we suggest facilitates the transition from the closed to the open form of RF2 (Fig. 5f, Supplementary Fig. 7d-i) and thereby enables placement of the GGQ motif within domain 3 (d3) of RF2 at the PTC of the ribosome.

\section{Discussion}

We have shown that Gram-positive bacteria, such as Bacillus, possess an RF-dependent ribosome rescue pathway, which had previously been known to occur only in Gram-negative bacteria (Fig. 6a). In this pathway in B. subtilis, BrfA plays a critical role in the hydrolytic release of the incomplete polypeptide from the non-stop stalled ribosomes. It does so by recruiting RF2 in a stopcodon-independent manner to the otherwise dead-end translation complex, as shown by our biochemical experiments using purified components. Our cryo-EM structure also reveals that BrfA recognizes the empty mRNA channel of a non-stop ribosome complex to recruit and stabilize the active (open) conformation of RF2 on the ribosome (Fig. 6b), in a similar but distinct manner to $\operatorname{ArfA}^{37-39,42-44}$ (Fig. 6c).

In vivo, the $b r f A$ deletion mutation exhibits a synthetic lethal phenotype when combined with CRISPRi-mediated knock-down or deletion of either SsrA or SmpB. Thus, BrfA is essential for growth in the absence of trans-translation activity. These findings answer the long-standing question of why the trans-translation system is not essential in B. subtilis, which lacks ArfA, ArfB and ArfT. Instead, BrfA is the alternative ribosome rescue factor in this organism and some other Bacillus species. The growth requirement of $B$. subtilis for BrfA and trans-translation is observed under normal growth conditions without the imposition of proteotoxic stresses. Thus, clearing of constitutively produced aberrant states of translation might be critical for cell survival, and BrfA and trans-translation components are the major players in this basic quality control. In this regard, the recently reported $\mathrm{RqcH}$ pathway, which adds a protein-degrading polyalanine sequence to the arrested polypeptide on the split $50 \mathrm{~S}$ subunit of the ribosome $\mathrm{e}^{50}$, appears to play a minor role in ribosome rescue under normal growth conditions, since $\mathrm{RqcH}$ is dispensable for trans-translation deficient strains to grow in the absence of stress. By contrast, $\mathrm{RqcH}$ appears to be required under more harsh conditions, such as high temperature and the presence of translation-disturbing drugs ${ }^{50}$.

During BrfA-mediated ribosome rescue, peptidyl-tRNA hydrolysis depends on an intact GGQ motif of RF2, indicating 
a

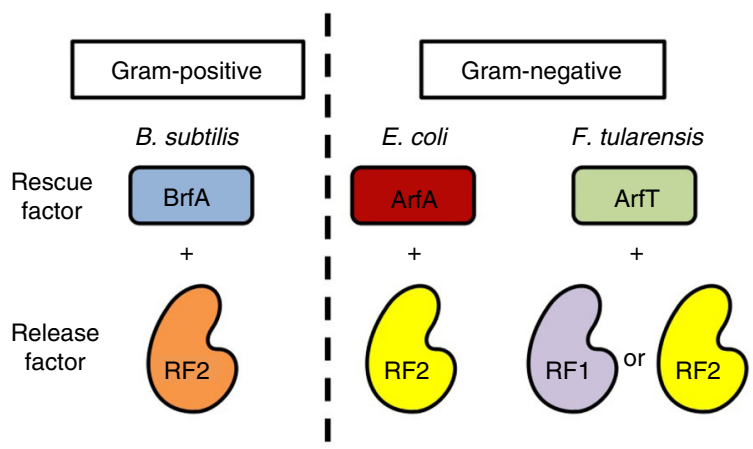

b

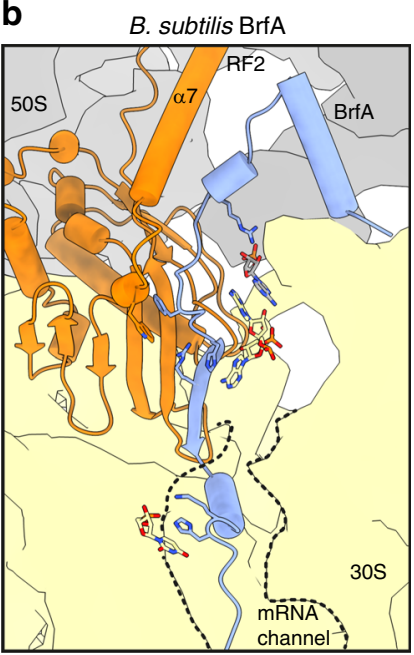

c

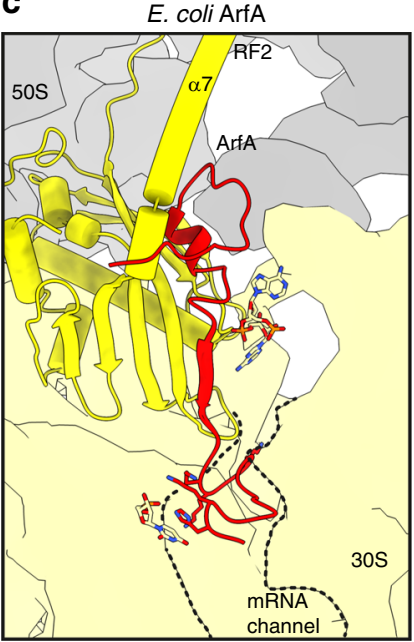

Fig. 6 RF-dependent ribosome rescue factors in bacteria. a Independent evolution of the RF-dependent ribosome rescue factors. ArfA and BrfA are unrelated in their amino acid sequences despite the same partner (RF2) specificity. ArfT forms a still distinct group and cooperates with either of RF1 or RF2. They show narrow distributions among bacterial species. b, c A schematic model highlighting the similarities and differences between (b) BrfA- and (c) ArfA-mediated ribosome rescue mechanism.

that RF2 plays the catalytic role. The role of BrfA is rather to recruit RF2 to the ns70S complex to initiate the events of stop codon-independent translation termination and thereby allow the subsequent ribosome recycling process. We used the Bs PURE system containing only a minimal set of essential translation components from E. coli together with B. subtilis ribosomes to recapitulate the rescue reaction in vitro, making it unlikely that other unknown cellular factors, especially those from B. subtilis, are required for the process. Moreover, we could show structurally that BrfA alone is sufficient to recruit and induce an active (open) conformation of BsRF2 on the non-stop ribosome complex. Taken together, we propose that BrfA is an RF-dependent ribosome rescue factor in Gram-positive bacteria, such as Bacillus (Fig. 6a).

In the process of canonical translation termination, stop codon recognition by the SPF motif of RF2 is a prerequisite for the process in which RF2 is accommodated into the A-site of the ribosome and adopting a catalytically active (open) conformation where the GGQ motif is directed into the PTC $40,48,51,52$. However, our structural data show that BrfA does not directly mimic the stop codon in the A-site (Fig. 5c), consistent with our observations that the stop codon recognition motif SPF is not required for ribosome rescue (Fig. $3 \mathrm{~b}$; see ref. ${ }^{16}$ for the similar situation in E. coli ArfA). Instead, the role of BrfA is to induce an open conformation of RF2, despite the absence of a stop codon.
BrfA appears to facilitate ribosome rescue using a different mechanism than reported previously for $\operatorname{ArfA}^{37-39,42-44}$. For example, during stop codon decoding, the switch loop Trp stacks directly upon A1492 of the 16S rRNA (Fig. 5d, Supplementary Fig. 7a), whereas during BrfA-mediated recycling, a series of stacking interactions appear to indirectly relay this informationspecifically, Phe31 of BrfA stacks upon the switch loop Trp318 of RF2, while His34 (and Arg36) of BrfA stacks upon A1492 (Fig. 5e, Supplementary 7b). By contrast, during ArfA-mediated rescue, the equivalent $\operatorname{Trp}$ residue inserts into a hydrophobic pocket created by ArfA and A1492 adopts a completely unrelated conformation (Supplementary Fig. 7c) $37-39,42-44$.

Perhaps the most dramatic difference between BrfA and ArfA is found in the N-terminal region, where BrfA contains two short a-helices that establish multiple interactions with $\mathrm{H} 69$ and $\mathrm{H} 71$ of the 23S rRNA, whereas the N-terminus of ArfA is unstructured and does not establish any interactions with the 50S subunit (Supplementary Fig. 5a-c). Despite this lack of sequence and structural homology, there are some common features between the two rescue systems: Firstly, both ArfA and BrfA (as well as SmpB and ArfB) appear to utilize positively charged C-terminal extensions to interact with the negatively charged 16S rRNA comprising the mRNA channel. Although the details of the interactions are unrelated, we identified a Lys-His (K49-H50 in B. subtilis) motif that is conserved between BrfA and ArfA, which is used to establish contacts to the backbone of nucleotide U534 of the 16S rRNA (Supplementary Fig. 5j-1). Secondly, both ArfA and BrfA contain short $\beta$-strands that augment the $\beta$-sheet in domain $2 / 4$ of RF2, which we presume is important to recruit RF2 to the ribosome (Supplementary Fig. $5 \mathrm{a}-\mathrm{c}$ ).

Here we show that BrfA works with RF2, but not RF1, reminiscent of the partner selectivity described previously for $\mathrm{ArfA}^{16}$ Analysis of the contacts between BrfA and RF2 within the BrfARF2-ns70S complex, and comparison with the sequence alignments between $B$. subtilis RF2 and RF1, indicated that there are two main regions in the RFs that are likely to be responsible for the selectivity of BrfA (Supplementary Fig. 8). These encompass residues within the $\beta$-sheet of domain $2 / 4$ of RF2 that are in proximity of Phe31 of BrfA. For example, in RF2 residues 197 and 216 are generally $\mathrm{Val}$ and Phe, respectively, whereas in RF1 the equivalent residues are replaced with the smaller Ala and Thr residues, respectively (Supplementary Fig. 8a). More dramatic, however, is the lack of sequence conservation between the switch loops of B. subtilis RF1 and RF2. In fact, the switch loop of RF1 is one residue longer than in RF2 (Supplementary Fig. 8b, d). We also note that the RF-dependent rescue factors show low interspecies compatibility (Fig. 3c). The incompatibility of BrfA to work with E. coli RF2 is not surprising given the relatively low sequence conservation observed within the switch region (Supplementary Fig. 8d). Moreover, sequence differences are also observed with $a$-helix 7 between $B$. subtilis and E. coli RF2 that could contribute to the interactions with BrfA (Supplementary Fig. 8c). Collectively, these differences suggest that even if BrfA could recruit $B$. subtilis RF1 or E. coli RF2 to the ns70S, the sequence differences in the switch loop are unlikely to stabilize the open conformation of RF2 on the ribosome. The lack of interspecies compatibility of BrfA (and ArfA) contrasts with other, more ubiquitous ribosome rescue factors. For example, $B$. subtilis tmRNA is functional in E. coli, albeit with lower efficiency ${ }^{25}$, and the human mitochondrial ArfB homolog, ICT1, is functional in C. crescentus and vice versa ${ }^{23}$. This low interspecies compatibility of the RF-dependent rescue factors reinforces the notion that these rescue systems emerged late in evolution.

It is crucial for the ribosome rescue factors not to intervene during normal translation, nor when translation is arrested by nascent polypeptides for regulatory purposes ${ }^{33}$. Our cryo-EM 
structure of the BrfA-RF2-ns70 reveals that BrfA uses its Cterminal region to monitor the vacancy of the ribosomal mRNA channel, a hallmark of the ribosomes stalled on the $3^{\prime}$ end of mRNA, and thereby discern non-stop translation complexes from those of ongoing translation ${ }^{42}$. Consistently, we have shown that neither BrfA, nor trans-translation, can resolve the MifMprogrammed elongation arrest complexes (Supplementary Fig. 1). In this case, the mRNA channel should remain occupied by the mifM mRNA. Also, the cryo-EM structures of the MifMstalled ribosomes reveal altered conformations of $23 \mathrm{~S}$ rRNA residues near the peptidyltransferase center (PTC) such that they block accommodation of aminoacyl-tRNAs or mimics thereof into the A-site ${ }^{53}$ explaining why MifM stalling is refractory to the BrfA-RF2 and the SmpB-tmRNA actions, too.

We have shown that $\operatorname{brfA}$ is transcribed as a non-stop mRNA because of the terminator sequence within the coding region. Thus, brfA itself is subject to a futile type of translation, producing a non-stop polypeptide that is extended by the SsrA tag sequence and rapidly eliminated by proteolysis. BrfA accumulates only when trans-translation is impaired, and in the form of peptidyl-tRNA. However, once some free BrfA product is generated by spontaneous hydrolysis of the BrfA peptidyl-tRNA, or by the presence of residual BrfA in the cell, it actively liberates in trans the BrfA peptide in a self-perpetuating manner. We have shown clearly using biochemical assays that a liberated form of the non-stop BrfA peptide (residues 1-62) is active in mediating the RF2-dependent peptidyl-tRNA hydrolysis.

Because BrfA is not effectively produced in trans-translation proficient cells, but induced strikingly upon dysfunction of transtranslation, it is likely to represent a secondary, back-up rescue system that compensates for defects in trans-translation. This scenario reinforces the notion that the proteolytic function characteristically associated with the SmpB-tmRNA system is not essential for growth, as reported previously ${ }^{13,14}$. Indeed, the GFPns non-stop product accumulates in the ssrA-deleted cell (Supplementary Fig. 1a), indicating that the products of the BrfA system are not necessarily toxic. Thus, the growth-essential roles of trans-translation and BrfA are in their ribosome recycling functions, rather than in the tagging-proteolysis that the BrfA system lacks. Cell viability would require a sufficient pool of the uncompromised ribosomes, which maintains the translation capacity of the cell. Whereas the two pathways share the essential function required for growth-supporting ribosome rescue, the proteolytic functions of the trans-translation and that of the $\mathrm{RqcH}$ tail-adding system could become more important under more severe stress conditions ${ }^{50}$.

The regulatory scheme of BrfA expression is strikingly similar to that elucidated for ArfA regulation in E. coli ${ }^{17,18}$; the $\operatorname{arfA}$ mRNA is also subject to RNase III-dependent cleavage and/or transcription termination such that ArfA only accumulates when trans-translation is defective. Assuming that BrfA and ArfA are evolutionarily unrelated, it is noteworthy that they employ a similar scheme of regulation. Convergent acquisition of such regulatory mechanisms may be more common for factors that have evolved recently and which have functions related to the firmly established and relatively rigid constituent of the cell, such as the ribosome and translation factors.

In summary, our study reveals that bacteria, both Gramnegative and Gram-positive, have RF-dependent mechanisms of ribosome rescue that allow for the stop-codon-independent liberation of the polypeptide from the ribosome on the non-stop mRNA (Fig. 6a). However, the crucial adapter proteins, ArfA, ArfT, and BrfA, are unrelated in amino acid sequence (Supplementary Fig. 2). The modes of their interactions with the catalytic RF partner(s) are also divergent, indicative of the tailored nature of their evolution in different species. It remains possible that many more factors in this category exist in different organisms. As the present study suggests that sequence similarity alone cannot be used to identify additional factors that exist in the domains of life, we need better strategies to address this question. Further studies on the generality and diversity of independently evolved rescue factors and their manner of interaction with the ribosome and translation factor would provide invaluable insights into regulatory mechanisms of translation processes in the cell.

\section{Methods}

Bacterial strains and plasmids. B. subtilis and E. coli strains, plasmids, DNA oligonucleotides used in this study are listed in Supplementary Tables 2, 3, 4 and 5 , respectively. The B. subtilis strains were derivatives of PY79 (wild-type; ref. ${ }^{54}$ ) and constructed by transformation that involves homologous recombination with plasmids listed in Supplementary Table 6. These plasmids carried an engineered $B$ subtilis gene to be integrated, which was flanked by sequences from the integration target loci, and were constructed by standard cloning methods including PCR, PrimeSTAR mutagenesis (Takara), and Gibson assembly ${ }^{55}$. The plasmid pCH747 was constructed by cloning a SphI-SpeI fragment of pCH735 into pyqjG21. Plasmids pCH735 and pyqjG21 were constructed as described ${ }^{35}$. Successful integration of a gene into the chromosome was accomplished by double crossing-over at the target loci. The resulting recombinant clones were checked for their antibioticresistance markers, including the absence of those originally present on the plasmid backbone, and inactivation of the amyE, lacA, or $\operatorname{thr} C$ target locus. The marker-less deletion mutants of $\operatorname{smp} B, y e s Z$, and lacA were constructed by excising the drug resistance gene cassette by the Cre-loxP system as described previously ${ }^{27}$ with some modification as follows. The $B$. subtilis strains were transformed with $\mathrm{pMK} 2$, a pLOSS*-based Ts plasmid harboring cre. The resulting strain was grown at $37^{\circ} \mathrm{C}$ overnight in LB agar medium supplemented with $1 \mathrm{mM}$ IPTG (isopropyl $\beta$-D-1thiogalactopyranoside) and $100 \mu \mathrm{g} / \mathrm{mL}$ spectinomycin to excise the drug marker flanked by $\operatorname{lox} \mathrm{P}^{27}$. The strain was then grown at $37^{\circ} \mathrm{C}$ overnight to drop off pMK2 on LB agar medium without spectinomycin. The absence of the drug resistance confirmed the absence of plasmid pMK2. The B. subtilis strain KFB792 was constructed by transformation of PY79 with a DNA fragment prepared by Gibson assembly with three PCR fragments, one of which was amplified from pCH1142 using a pair of primers SP89/SP90, and the other two of which were amplified from PY79 genomic DNA using pairs of primers SP91/SP92 and SP93/SP94, respectively.

Growth conditions and general procedures. For western blotting in Fig. 2c, $B$. subtilis cells were cultured at $37^{\circ} \mathrm{C}$ in LB medium with or without $0.5 \%$ xylose unti $\mathrm{OD}_{600}$ reached $\sim 0.5$. Bacterial culture $(1 \mathrm{~mL})$ was treated with $5 \%$ trichloroacetic acid (TCA), and precipitates formed were washed with $0.75 \mathrm{~mL}$ of $1 \mathrm{M}$ Tris- $\mathrm{HCl}$ ( $\mathrm{pH}$ 8.0) and resuspended in $50 \mu \mathrm{L}$ of buffer L ( $33 \mathrm{mM}$ Tris- $\mathrm{HCl}, 1 \mathrm{mM}$ EDTA, pH 8.0 ) containing $1 \mathrm{mg} / \mathrm{mL}$ lysozyme, followed by incubation at $37^{\circ} \mathrm{C}$ for $10 \mathrm{~min}$. Proteins were then solubilized with an equal volume of $2 \times$ SDS-loading buffer containing $5 \mathrm{mM}$ dithiothreitol (DTT) with incubation at $65^{\circ} \mathrm{C}$ for $5 \mathrm{~min}$ and subjected to SDS-PAGE and immunoblotting. One hundred microliters portions of the bacterial cultures were used for assay of $\beta$-galactosidase activity, presented in Fig. $2 \mathrm{~d}$ as follows and also as described previously ${ }^{31}$. The cultures were transferred to individual wells of another 96-well plate for a Thermo Scientific Multiskan Go microplate spectrophotometer and $\mathrm{OD}_{600}$ was recorded. Cells were then lysed by adding $50 \mu \mathrm{L}$ of Y-PER reagent (Thermo Scientific) and incubating for $20 \mathrm{~min}$ at room temperature. Thirty microliters of $o$-nitrophenyl- $\beta$-D-galactopyranoside (ONPG) in Z-buffer (60 mM Na $2 \mathrm{HPO}_{4}, 40 \mathrm{mM} \mathrm{NaH}_{2} \mathrm{PO}_{4}, 10 \mathrm{mM} \mathrm{KCl}, 1 \mathrm{mM}$ $\mathrm{MgSO}_{4}, 38 \mathrm{mM} \beta$-mercaptoethanol) was added to each well. The reaction solution was mixed thoroughly and then $\mathrm{OD}_{420}$ and $\mathrm{OD}_{550}$ were measured every 5 min over $60 \mathrm{~min}$ at $28^{\circ} \mathrm{C}$. Arbitrary units [AU] of $\beta$-galactosidase activity were calculated by the formula $\left[\left(1000 \times V_{420}-1.3 \times V_{550}\right) / \mathrm{OD}_{600}\right]$, where $V_{420}$ and $V_{550}$ are the firstorder rate constants, $\mathrm{OD}_{420} / \mathrm{min}$ and $\mathrm{OD}_{550} / \mathrm{min}$, respectively.

Synthetic lethal screening using the BKE strain library. To isolate B. subtilis mutants whose viability depends on trans-translation, we used the BKE library (a collection of single-gene knockout mutants covering the 3968 non-essential genes, which had been disrupted by replacement with the erythromycin resistance marker) as the source of gene knockouts. We pooled the BKE strains and prepared a genomic DNA mixture using Wizard genome DNA purification kit (Promega). We

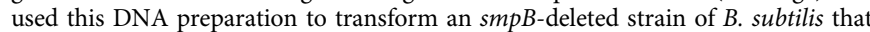
harbored a rescue plasmid carrying $s m p B^{+}$and $l a c Z^{+}$(pNAB1286), which was constructed from pLOSS* with a temperature sensitive (Ts) replication system. Transformant mixture was then incubated at $50^{\circ} \mathrm{C}$ overnight to segregate out the Ts plasmid from bacteria that did not need the rescue plasmid, followed by further growth at $37^{\circ} \mathrm{C}$ overnight and plating on LB agar containing $40 \mu \mathrm{g} / \mathrm{mL} \mathrm{X-Gal}(5$ bromo-4-chloro-3-indolyl $\beta$-D-galactopyranoside), $1 \mathrm{mM} \mathrm{IPTG,} 12.5 \mu \mathrm{g} / \mathrm{mL}$ lincomycin, and $1 \mu \mathrm{g} / \mathrm{mL}$ erythromycin. We picked up blue $\left(\operatorname{lac} Z^{+}\right)$colonies to obtain transformants that retained the $s m p B^{+}-l a c Z^{+}$rescue plasmid even after the hightemperature incubation and prepared chromosomal DNA from them. Genes that had been disrupted by the erythromycin resistance marker were determined by 
PCR amplification and DNA sequencing of the mutant-specific barcode sequence, using appropriate primers ${ }^{27}$.

CRISPR interference. The CRISPRi was performed as described previously ${ }^{56}$ with some modification. A gene encoding dCas9 under the xylose-inducible promoter was integrated into the lacA site on the chromosome of a brfA-deleted B. subtilis strain, into which an $s s r A$-targeted or an $s m p B$-targeted sgRNA gene under a constitutive promoter was further integrated at the $a m y E$ site. The guide sequences for CRISPRi were designed on the basis of information provided by the previous study $^{56}$ as well as CHOPCHOP, a web tool for the CRISPR/Cas9 experiments ${ }^{57,58}$. The target gene knock-down was induced by addition of $1 \%$ xylose.

Protein purification. Hexahistidine-tagged proteins (B. subtilis $\mathrm{BrfA}^{2}-\mathrm{His}_{6}$, RF1$\mathrm{His}_{6}$, RF2-His ${ }_{6}$, E. coli $\mathrm{His}_{6}$-ArfA60, and their derivatives) were expressed in E. coli strain BL21(DE3) from the pET28b-based plasmid. E. coli cells were grown in LBkanamycin $(25 \mu \mathrm{g} / \mathrm{mL})$ medium. At a mid-log phase, IPTG (final concentration, $1 \mathrm{mM}$ ) was added, and cells were grown for an additional $3 \mathrm{~h}$ to express and accumulate the target protein. Cells were then harvested, washed with ice-cold $50 \mathrm{mM}$ HEPES-NaOH ( $\mathrm{pH}$ 7.6) buffer, and stored at $-80^{\circ} \mathrm{C}$. They were suspended in binding buffer (50 mM HEPES- $\mathrm{KOH} \mathrm{pH} 7.6,5 \mathrm{mM}$ imidazole, $300 \mathrm{mM} \mathrm{NaCl}$, $1 \mathrm{mg} / \mathrm{mL}$ Pefabloc) and disrupted by passing through a microfluidizer LV1 (Microfluidics) at 16,000 psi three times. After removal of debris by centrifugation $\left(4^{\circ} \mathrm{C}, 15,000\right.$ r.p.m. for $\left.15 \mathrm{~min}\right)$, Ni-NTA agarose was added to the sample, which was then incubated at $4{ }^{\circ} \mathrm{C}$ for $1 \mathrm{~h}$. The Ni-NTA agarose was loaded on a spin column and washed seven times with wash buffer (50 mM HEPES-KOH pH.7.6, $20 \mathrm{mM}$ imidazole, $0.1 \%$ Triton-X). Protein was eluted with the elution buffer (20 mM HEPES-KOH pH.7.6, $300 \mathrm{mM} \mathrm{NaCl}, 300 \mathrm{mM}$ imidazole). Purified RFs were dialyzed against dialysis buffer A (50 mM HEPES-KOH pH.7.6, $100 \mathrm{mM}$ potassium acetate, $1 \mathrm{mM}$ DTT, $30 \%$ glycerol). Purified BrfA62-His ${ }_{6}$ and $\mathrm{His}_{6}$-ArfA (2-60) were dialyzed against dialysis buffer B (50 mM HEPES-KOH pH.7.6, $100 \mathrm{mM}$ potassium acetate, $1 \mathrm{mM}$ DTT, $300 \mathrm{mM} \mathrm{NaCl}, 30 \%$ glycerol).

For the structural analysis, the wild-type B. subtilis RF2 and variant RF2-GGP protein were expressed from pET11a vectors incorporating a C-terminal hexahistidine tag $\left(\mathrm{His}_{6}\right)$ for purification and detection purposes. The inactive RF2GGP mutant was generated by site-directed mutagenesis. The wild-type RF2 and RF2-GGP proteins were over-expressed in E. coli BL21 (DE3) at $37^{\circ} \mathrm{C}$ for $1.5 \mathrm{~h}$ after induction with $1 \mathrm{mM}$ IPTG. Cells were collected and the pellet was resuspended in lysis buffer $\left(50 \mathrm{mM} \mathrm{NaH}_{2} \mathrm{PO}_{4}, 300 \mathrm{mM} \mathrm{NaCl}, 5 \mathrm{mM}\right.$ imidazole, $\mathrm{pH}$ 7.5). Lysis was performed using a microfluidizer (Microfluidics M-110L) by passing cells three times (at 18,000 psi). The cell debris was removed upon centrifugation and the proteins were purified from the supernatant by His-tag affinity chromatography using Ni-NTA agarose beads (Clontech). The bound proteins were washed with lysis buffer containing $10 \mathrm{mM}$ imidazole and then eluted with lysis buffer containing $250 \mathrm{mM}$ imidazole. The proteins RF2, RF2-GGP, and BrfA62-His ${ }_{6}$ were purified by size-exclusion chromatography using HiLoad 16/600 Superdex 75 (GE Life Sciences) in gel filtration buffer (50 mM HEPES, pH 7.4, $50 \mathrm{mM} \mathrm{KCl}, 100 \mathrm{mM} \mathrm{NaCl}, 2 \%$ glycerol, $5 \mathrm{mM} \beta$-mercaptoethanol). The proteins were concentrated using Amicon Ultracel-30 Centrifugal Filter Units (Merck Millipore) for wild-type RF2 and RF2-GGP and Ultracel-3 for BrfA62-His 6 hereafter referred to as BrfA.

\section{In vitro translation using PURE system. The E. coli-based coupled} transcription-translation system with purified components (PUREfrex 1.0; GeneFrontier) was used for in vitro translation as described previously ${ }^{29-31}$ with some modifications. To maximize transcription, we added $2.5 \mathrm{U} / \mu \mathrm{L}$ of T7 RNA polymerase (Takara) further to the reaction mixture. Whereas the original reaction mixture, referred to as $E c$ PURE system, contained the E. coli ribosome, we also used the Bs hybrid PURE system containing the B. subtilis ribosomes at a final concentration of $1 \mu \mathrm{M}$. Unless otherwise noted, we omitted RF1, RF2, and RF3 from the reaction. However, we included purified RF or its derivatives derived either from B. subtilis or E. coli at a final concentration of $1 \mu \mathrm{M}$ as indicated in each experiment. E. coli RFs were purchased from GeneFrontier. Purified BrfA or ArfA was added to the final concentration of $1 \mu \mathrm{M}$ when indicated. The reaction was primed with an appropriate DNA fragment prepared by PCR (Supplementary Table 7) and allowed to continue at $37^{\circ} \mathrm{C}$ for $20 \mathrm{~min}$. Samples were then mixed with the same volume of $2 \times$ SDS-PAGE loading buffer. When indicated, they were further treated with $0.2 \mathrm{mg} / \mathrm{mL}$ RNase A (Promega) at $37^{\circ} \mathrm{C}$ for $15 \mathrm{~min}$ before electrophoresis. Samples for SDS-PAGE were heated at $65^{\circ} \mathrm{C}$ for $5 \mathrm{~min}$, separated by $10 \%$ wide range gel (Nacalai Tesque) ${ }^{31}$ and transferred on to a PVDF membrane. Translation products were detected by immunoblotting using anti-GFP (A6455; Thermo) or anti-DYKDDDDK (anti-FLAG tag; Wako) as described previously ${ }^{31}$. Images were obtained and analyzed using an Amersham Imager 600 (GE Healthcare) luminoimager. Uncropped immunoblotting data are shown in the Source Data file.

Generation of BrfA-RF2-ns70S complex. Generation of the BrfA-RF2-ns70S complex was similar to that previously described for the ArfA-RF2-70S complex ${ }^{39}$. Briefly, the truncated nlpD template containing an $\mathrm{N}$-terminal $\mathrm{His}_{6}$ and HA-tag was first amplified from pET21b-r1nlpD using T7-promotor and nucleotides 133-159 of nlpD as reverse primer. Following PCR purification via spin column (Qiagen), in vitro translation (PURExpress, NEB 6800) was started by adding the truncated $n l p D$ PCR product at $37^{\circ} \mathrm{C}$ for $20 \mathrm{~min}$, shaking at 1000 r.p.m. The ribosomes were first isolated from the in vitro reaction mix by centrifugation through a sucrose cushion (50 mM HEPES-KOH pH 7.2, $250 \mathrm{mM}$ potassium acetate, $25 \mathrm{mM}$ magnesium acetate, $750 \mathrm{mM}$ sucrose, $0.1 \%$ DDM) for $180 \mathrm{~min}$ at $72,000 \times g$ using a TLA120.2 rotor (Beckman Coulter). The pellet was resuspended in buffer B250 (50 mM HEPES-KOH pH 7.2, $250 \mathrm{mM}$ potassium acetate, $25 \mathrm{mM}$ magnesium acetate, $0.1 \% \mathrm{DDM}$ ) and the ns $70 \mathrm{~S}$ complex was isolated using Talon cobalt-chelate affinity resin (Clontech). ns70S complex bound to the Talon matrix by the $\mathrm{N}$-terminal $\mathrm{His}_{6}$ tag of NlpD was washed with buffer B500 (50 mM HEPES$\mathrm{KOH} \mathrm{pH} 7.2,500 \mathrm{mM}$ potassium acetate, $25 \mathrm{mM}$ magnesium acetate, $0.1 \% \mathrm{DDM}$ ) and eluted using buffer B250i (50 mM HEPES-KOH pH 7.2, $250 \mathrm{mM}$ potassium acetate, $25 \mathrm{mM}$ magnesium acetate, $250 \mathrm{mM}$ imidazole, $0.1 \% \mathrm{DDM})$. The eluted ns70S complex was loaded onto a linear sucrose gradient $(10-40 \%(\mathrm{w} / \mathrm{v})$ sucrose in B250 buffer) for $18 \mathrm{~h}$ at $43,000 \times g$ in a SW28 rotor (Beckman Coulter). The isolated $70 \mathrm{~S}$ peak was pelleted by centrifugation for $3 \mathrm{~h}$ at $139,000 \times g$ using a Ti70.1 rotor (Beckman Coulter). The pellet was resuspended in ns70S complex buffer (50 mM HEPES $\mathrm{pH} 7.2,250 \mathrm{mM}$ potassium acetate, $10 \mathrm{mM}$ magnesium acetate, $0.05 \% \mathrm{DDM})$. The purified non-stop ribosome complex was then incubated together with a 10× excess of BrfA and RF2-GGP mutant for $5 \mathrm{~min}$ at $37^{\circ} \mathrm{C}$ before being applied to cryo-EM grids.

Cryo-EM and single-particle reconstruction. Three microliters $\left(4.5 \mathrm{OD}_{260 \mathrm{~nm}}\right.$ per $\mathrm{mL}$ ) of BrfA-RF2-ns70S complex was applied to $2 \mathrm{~nm}$ pre-coated Quantifoil R3/3 holey carbon supported grids and vitrified using the Vitrobot Mark IV (FEI, Holland). Data collection was performed using EM-TOOLS (TVIPS GmbH) on a Titan Krios transmission electron microscope equipped with a Falcon III direct electron detector (FEI, Holland) at $300 \mathrm{keV}$ at a pixel size of $1.065 \AA$ and a defocus range of $0.4-2.2 \mu \mathrm{m}$. Forty frames (dose per frame of $2 e^{-} \AA^{-2}$ ) were aligned using Motion Correction software ${ }^{59}$. Power spectra and defocus values were determined using the GCTF software ${ }^{60}$. Micrographs showing thon rings beyond $3.2 \AA$ were manually inspected for good areas and automatic particle picking was performed using the Gautomatch software (http://www.mrclmb.cam.ac.uk/kzhang/). Single particles were then imported and processed in Relion 3 (ref. ${ }^{61}$ ). In total, 514,119 particles were first subjected to $2 \mathrm{D}$ classification (60 classes for 100 rounds) and 389,381 particles showing ribosome-like features were then selected for 3D refinement using an E. coli 70 S ribosome as a reference structure (Supplementary Fig. 4a). 3D classification was then performed, resulting in 317,095 particles containing P-site tRNA and RF2 (Supplementary Fig. 4b) that were further selected for focus sorting on the RF2 (Supplementary Fig. 4c). Focus sorting yielded a major population of 154,405 particles containing stoichiometric amounts of BrfA, P-site tRNA, RF2, which after CTF-refinement and a final round of 3D refinement produced a final cryo-EM reconstruction with an average resolution of $3.06 \AA$ according to $\mathrm{FSC}_{0.143}$ criterion (Supplementary Fig. $4 \mathrm{~d}$ ). The final cryo-EM maps were sharpened by dividing the maps by the modulation transfer function of the detector and applying an automatically determined negative B factor in Relion 3 (ref. ${ }^{61}$ ). The final cryo-EM map was also filtered according to local resolution using SPHIRE ${ }^{62}$.

Molecular modeling of the BrfA-RF2-ns70S complex. The molecular model for the ribosomal proteins and rRNA core was based on the molecular model from the recent cryo-EM reconstructions of the E. coli 70 S ribosome (PDB ID $6 \mathrm{H}_{4} \mathrm{~N}^{63}$ and $5 \mathrm{MGP}^{39}$ ). The models were rigid body fitted into the cryo-EM density map using UCSF Chimera followed by refinement in Coot $^{64}$. Proteins L1, L10, L11 protein, and the L7/L12 stalk were not included in the final model due to the poor quality of density in the final cryo-EM map. For B. subtilis RF2, a homology model was generated using HHPred ${ }^{65}$ based on an E. coli RF2 template (PDB ID 5MGP ${ }^{39}$ ). Domains 1 and 3 of RF2 were less well-resolved due to high flexibility (Supplementary Fig. 4e) and therefore only the backbone was modeled. Residues 2-55 of BrfA were built de novo using an HHPred model ${ }^{65}$ as an initial starting point to determine the placement of the central helical regions. The complete atomic model of the BrfA-RF2-ms70S complex was manually adjusted using $\operatorname{Coot}^{64}$ and refined with phenix real_space_refine for cryo- $\mathrm{EM}^{64}$ using restraints obtained by phenix secondary_structure_restraints ${ }^{64}$. The model refinement and statistics of the refined model were obtained using MolProbity ${ }^{66}$ (Supplementary Table 1).

Figure preparation. Figures showing electron densities and atomic models were generated using either UCSF Chimera, UCSF ChimeraX ${ }^{67}$ or PyMol (Version 1.8 Schrödinger). The comparison of BrfA with full-length mRNA (PDB ID 4V5E ${ }^{48}$ ), ArfA (PDB ID 5MGP $39,5 \mathrm{MDV}^{37}, 5 \mathrm{UPF}^{44}$ ), RF2 $2_{\text {closed }}$ (PDB ID $1 \mathrm{GQE}$ ), and RF2 $2_{\text {stop }}$ (PDB ID 4V5E ${ }^{48}$ ) was obtained by alignment of the $16 \mathrm{~S}$ rRNAs from the respective structures using PyMol (Version 1.8 Schrödinger).

Reporting summary. Further information on research design is available in the Nature Research Reporting Summary linked to this article. 


\section{Data availability}

The cryo-EM map of the BrfA-RF2-ns70S complex is available through the EMDB code EMD-10353 and the associated molecular model is deposited in the Protein Data Bank with the entry code 6SZS (https://doi.org/10.2210/pdb6SZS/pdb). The data that support the findings of this study are available from the corresponding authors on request. The source data underlying Figs $2 \mathrm{c}, \mathrm{d}$ and $3 \mathrm{a}-\mathrm{c}$ and Supplementary Figs $1 \mathrm{a}, 1 \mathrm{~b}$ and 3 are provided as a Source Data file.

Received: 2 August 2019; Accepted: 7 November 2019;

Published online: 27 November 2019

\section{References}

1. Youngman, E. M., McDonald, M. E. \& Green, R. Peptide release on the ribosome: mechanism and implications for translational control. Annu. Rev. Microbiol. 62, 353-373 (2008).

2. Petry, S., Weixlbaumer, A. \& Ramakrishnan, V. The termination of translation. Curr. Opin. Struct. Biol. 18, 70-77 (2008).

3. Ito, K. et al. Nascentome analysis uncovers futile protein synthesis in Escherichia coli. PLoS ONE 6, e28413 (2011).

4. Buskirk, A. R. \& Green, R. Ribosome pausing, arrest and rescue in bacteria and eukaryotes. Philos. Trans. R. Soc. Lond. B Biol. Sci. 372, https://doi.org/ 10.1098/rstb.2016.0183 (2017).

5. Keiler, K. C. \& Feaga, H. A. Resolving nonstop translation complexes is a matter of life or death. J. Bacteriol. 196, 2123-2130 (2014).

6. Inada, T. The ribosome as a platform for mRNA and nascent polypeptide quality control. Trends Biochem. Sci. 42, 5-15 (2017).

7. Joazeiro, C. A. P. Ribosomal stalling during translation: providing substrates for ribosome-associated protein quality control. Annu. Rev. Cell Dev. Biol. 33, 343-368 (2017).

8. Himeno, H., Nameki, N., Kurita, D., Muto, A. \& Abo, T. Ribosome rescue systems in bacteria. Biochimie 114, 102-112 (2015).

9. Giudice, E. \& Gillet, R. The task force that rescues stalled ribosomes in bacteria. Trends Biochem. Sci. 38, 403-411 (2013).

10. Keiler, K. C., Waller, P. R. \& Sauer, R. T. Role of a peptide tagging system in degradation of proteins synthesized from damaged messenger RNA. Science 271, 990-993 (1996).

11. Karzai, A. W., Susskind, M. M. \& Sauer, R. T. SmpB, a unique RNA-binding protein essential for the peptide-tagging activity of SsrA (tmRNA). EMBO J. 18, 3793-3799 (1999).

12. Brunel, R. \& Charpentier, X. Trans-translation is essential in the human pathogen Legionella pneumophila. Sci. Rep. 6, 37935 (2016).

13. Thibonnier, M., Thiberge, J. M. \& De Reuse, H. Trans-translation in Helicobacter pylori: essentiality of ribosome rescue and requirement of protein tagging for stress resistance and competence. PLoS ONE 3, e3810 (2008).

14. Huang, C., Wolfgang, M. C., Withey, J., Koomey, M. \& Friedman, D. I. Charged tmRNA but not tmRNA-mediated proteolysis is essential for Neisseria gonorrhoeae viability. EMBO J. 19, 1098-1107 (2000).

15. Chadani, Y. et al. Ribosome rescue by Escherichia coli ArfA (YhdL) in the absence of trans-translation system. Mol. Microbiol. 78, 796-808 (2010).

16. Chadani, Y., Ito, K., Kutsukake, K. \& Abo, T. ArfA recruits release factor 2 to rescue stalled ribosomes by peptidyl-tRNA hydrolysis in Escherichia coli. Mol. Microbiol. 86, 37-50 (2012).

17. Chadani, Y. et al. trans-translation-mediated tight regulation of the expression of the alternative ribosome-rescue factor ArfA in Escherichia coli. Genes Genet. Syst. 86, 151-163 (2011).

18. Schaub, R. E., Poole, S. J., Garza-Sanchez, F., Benbow, S. \& Hayes, C. S. Proteobacterial ArfA peptides are synthesized from non-stop messenger RNAs. J. Biol. Chem. 287, 29765-29775 (2012).

19. Chadani, Y., Ono, K., Kutsukake, K. \& Abo, T. Escherichia coli YaeJ protein mediates a novel ribosome-rescue pathway distinct from SsrA- and ArfAmediated pathways. Mol. Microbiol. 80, 772-785 (2011).

20. Handa, Y., Inaho, N. \& Nameki, N. YaeJ is a novel ribosome-associated protein in Escherichia coli that can hydrolyze peptidyl-tRNA on stalled ribosomes. Nucleic Acids Res. 39, 1739-1748 (2011).

21. Gagnon, M. G., Seetharaman, S. V., Bulkley, D. \& Steitz, T. A. Structural basis for the rescue of stalled ribosomes: structure of YaeJ bound to the ribosome. Science 335, 1370-1372 (2012).

22. Feaga, H. A., Viollier, P. H. \& Keiler, K. C. Release of nonstop ribosomes is essential. MBio 5, e01916 (2014).

23. Feaga, H. A., Quickel, M. D., Hankey-Giblin, P. A. \& Keiler, K. C. Human cells require non-stop ribosome rescue activity in mitochondria. PLoS Genet. 12, e1005964 (2016).

24. Goralski, T. D. P., Kirimanjeswara, G. S. \& Keiler, K. C. A new mechanism for ribosome rescue can recruit RF1 or RF2 to nonstop ribosomes. MBio 9, https://doi.org/10.1128/mBio.02436-18 (2018).
25. Muto, A. et al. Requirement of transfer-messenger RNA for the growth of Bacillus subtilis under stresses. Genes Cells 5, 627-635 (2000).

26. Claessen, D. et al. Control of the cell elongation-division cycle by shuttling of PBP1 protein in Bacillus subtilis. Mol. Microbiol. 68, 1029-1046 (2008).

27. Koo, B. M. et al. Construction and analysis of two genome-scale deletion libraries for Bacillus subtilis. Cell Syst. 4, 291-305 e297 (2017).

28. Qi, L. S. et al. Repurposing CRISPR as an RNA-guided platform for sequencespecific control of gene expression. Cell 152, 1173-1183 (2013).

29. Chiba, S. et al. Recruitment of a species-specific translational arrest module to monitor different cellular processes. Proc. Natl. Acad. Sci. USA 108, 6073-6078 (2011).

30. Shimizu, Y. et al. Cell-free translation reconstituted with purified components. Nat. Biotechnol. 19, 751-755 (2001).

31. Fujiwara, K., Ito, K. \& Chiba, S. MifM-instructed translation arrest involves nascent chain interactions with the exterior as well as the interior of the ribosome. Sci. Rep. 8, 10311 (2018).

32. Ito, K., Uno, M. \& Nakamura, Y. A tripeptide 'anticodon' deciphers stop codons in messenger RNA. Nature 403, 680-684 (2000).

33. Ito, K. \& Chiba, S. Arrest peptides: cis-acting modulators of translation. Annu. Rev. Biochem. 82, 171-202 (2013).

34. Ito, K., Mori, H. \& Chiba, S. Monitoring substrate enables real-time regulation of a protein localization pathway. FEMS Microbiol. Lett. 365, https://doi.org/ 10.1093/femsle/fny109 (2018).

35. Chiba, S., Lamsa, A. \& Pogliano, K. A ribosome-nascent chain sensor of membrane protein biogenesis in Bacillus subtilis. EMBO J. 28, 3461-3475 (2009).

36. Chiba, S. \& Ito, K. MifM monitors total YidC activities of Bacillus subtilis, including that of YidC2, the target of regulation. J. Bacteriol. 197, 99-107 (2015).

37. James, N. R., Brown, A., Gordiyenko, Y. \& Ramakrishnan, V. Translational termination without a stop codon. Science 354, 1437-1440 (2016).

38. Zeng, F. et al. Structural basis of co-translational quality control by ArfA and RF2 bound to ribosome. Nature 541, 554-557 (2017).

39. Huter, P. et al. Structural basis for ArfA-RF2-mediated translation termination on mRNAs lacking stop codons. Nature 541, 546-549 (2017).

40. Korostelev, A. et al. Crystal structure of a translation termination complex formed with release factor RF2. Proc. Natl. Acad. Sci. USA 105, 19684-19689 (2008).

41. Santos, N., Zhu, J., Donohue, J. P., Korostelev, A. A. \& Noller, H. F. Crystal structure of the $70 \mathrm{~S}$ ribosome bound with the Q253P mutant form of release factor RF2. Structure 21, 1258-1263 (2013).

42. Huter, P., Muller, C., Arenz, S., Beckert, B. \& Wilson, D. N. Structural basis for ribosome rescue in bacteria. Trends Biochem. Sci. 42, 669-680 (2017).

43. Ma, C. et al. Mechanistic insights into the alternative translation termination by ArfA and RF2. Nature 541, 550-553 (2017).

44. Demo, G. et al. Mechanism of ribosome rescue by ArfA and RF2. Elife 6, e23687 (2017)

45. Shimizu, Y. ArfA recruits RF2 into stalled ribosomes. J. Mol. Biol. 423, 624-631 (2012).

46. Kurita, D., Chadani, Y., Muto, A., Abo, T. \& Himeno, H. ArfA recognizes the lack of mRNA in the mRNA channel after RF2 binding for ribosome rescue. Nucleic Acids Res. 42, 13339-13352 (2014).

47. Zeng, F. \& Jin, H. Peptide release promoted by methylated RF2 and ArfA in nonstop translation is achieved by an induced-fit mechanism. RNA 22, 49-60 (2016).

48. Weixlbaumer, A. et al. Insights into translational termination from the structure of RF2 bound to the ribosome. Science 322, 953-956 (2008).

49. Zhou, J., Korostelev, A., Lancaster, L. \& Noller, H. F. Crystal structures of 70S ribosomes bound to release factors RF1, RF2 and RF3. Curr. Opin. Struct. Biol. 22, 733-742 (2012).

50. Lytvynenko, I. et al. Alanine tails signal proteolysis in bacterial ribosomeassociated quality control. Cell 178, 76-90 e22 (2019).

51. Laurberg, M. et al. Structural basis for translation termination on the $70 \mathrm{~S}$ ribosome. Nature 454, 852-857 (2008).

52. Korostelev, A., Zhu, J., Asahara, H. \& Noller, H. F. Recognition of the amber UAG stop codon by release factor RF1. EMBO J. 29, 2577-2585 (2010).

53. Sohmen, D. et al. Structure of the Bacillus subtilis $70 \mathrm{~S}$ ribosome reveals the basis for species-specific stalling. Nat. Commun. 6, 6941 (2015).

54. Youngman, P., Perkins, J. B. \& Losick, R. A novel method for the rapid cloning in Escherichia coli of Bacillus subtilis chromosomal DNA adjacent to Tn917 insertions. Mol. Gen. Genet. 195, 424-433 (1984).

55. Gibson, D. G. et al. Enzymatic assembly of DNA molecules up to several hundred kilobases. Nat. Methods 6, 343-345 (2009).

56. Peters, J. M. et al. A comprehensive, CRISPR-based functional analysis of essential genes in bacteria. Cell 165, 1493-1506 (2016).

57. Montague, T. G., Cruz, J. M., Gagnon, J. A., Church, G. M. \& Valen, E. CHOPCHOP: a CRISPR/Cas9 and TALEN web tool for genome editing. Nucleic Acids Res. 42, W401-W407 (2014). 
58. Labun, K., Montague, T. G., Gagnon, J. A., Thyme, S. B. \& Valen, E. CHOPCHOP v2: a web tool for the next generation of CRISPR genome engineering. Nucleic Acids Res. 44, W272-W276 (2016).

59. $\mathrm{Li}, \mathrm{X}$. et al. Electron counting and beam-induced motion correction enable near-atomic-resolution single-particle cryo-EM. Nat. Methods 10, 584-590 (2013).

60. Zhang, K. Gctf: real-time CTF determination and correction. J. Struct. Biol. 193, 1-12 (2016).

61. Zivanov, J. et al. New tools for automated high-resolution cryo-EM structure determination in RELION-3. Elife 7, e42166 (2018).

62. Moriya, T. et al. High-resolution single particle analysis from electron cryomicroscopy images using SPHIRE. J. Vis. Exp., https://doi.org/10.3791/55448 (2017).

63. Beckert, B. et al. Structure of a hibernating 100 S ribosome reveals an inactive conformation of the ribosomal protein S1. Nat. Microbiol 3, 1115-1121 (2018).

64. Adams, P. D. PHENIX: a comprehensive Python-based system formacromolecular structure solution. Acta Crystallogr. D Biol. Crystallogr. 66 , 213-221 (2010).

65. Soding, J., Biegert, A. \& Lupas, A. N. The HHpred interactive server for protein homology detection and structure prediction. Nucleic Acids Res. 33 W244-W248 (2005).

66. Chen, V. B. et al. MolProbity: all-atom structure validation for macromolecular crystallography. Acta Crystallogr. D Biol. Crystallogr. 66, 12-21 (2010).

67. Goddard, T. D. et al. UCSF ChimeraX: meeting modern challenges in visualization and analysis. Protein Sci. 27, 14-25 (2018).

\section{Acknowledgements}

We thank Dr. Hyouta Himeno of Hirosaki University for the B. subtilis ssrA mutant strain; Dr. Jiři Nováček (CEITEC, Brno, Czech Republic) for help with high-resolution cryo-EM data collection; Dr. Allen Buskirk for discussion; and Yuko Sumino, Saori Amano, Sakika Otani, Toru Irie, S. Rieder, and C. Ungewickell for expert technical support. We are also thankful to the National BioResource Project for providing the BKE strain library. This work was supported by grants from MEXT and JSPS Grant-in-Aid for Scientific Research (Grant No. 25291006 and 16H04788 to S.C., 26116008 to S.C. and K.I., 19K16044 to K.F.) and the Deutsche Forschungsgemeinschaft (SPP1879-WI3285/5-2 to D.N.W.).

\section{Author contributions}

N.S.-C., K.F., and S.C. performed biochemical experiments and C.M., B.B., and D.N.W. performed structural experiments. N.S.-C., K.F., K.I., S.C., C.M., B.B., and D.N.W. designed experiments, analyzed data, and were involved in writing the manuscript.

\section{Competing interests}

The authors declare no competing interests.

\section{Additional information}

Supplementary information is available for this paper at https://doi.org/10.1038/s41467019-13408-7.

Correspondence and requests for materials should be addressed to D.N.W. or S.C.

Peer review information Nature Communications thanks the anonymous reviewers for their contribution to the peer review of this work. Peer reviewer reports are available.

Reprints and permission information is available at http://www.nature.com/reprints

Publisher's note Springer Nature remains neutral with regard to jurisdictional claims in published maps and institutional affiliations.

(c) Open Access This article is licensed under a Creative Commons Attribution 4.0 International License, which permits use, sharing, adaptation, distribution and reproduction in any medium or format, as long as you give appropriate credit to the original author(s) and the source, provide a link to the Creative Commons license, and indicate if changes were made. The images or other third party material in this article are included in the article's Creative Commons license, unless indicated otherwise in a credit line to the material. If material is not included in the article's Creative Commons license and your intended use is not permitted by statutory regulation or exceeds the permitted use, you will need to obtain permission directly from the copyright holder. To view a copy of this license, visit http://creativecommons.org/ licenses/by/4.0/.

(C) The Author(s) 2019 